\title{
REAL TIME DETECTION OF CACHE-BASED SIDE-CHANNEL ATTACKS USING HARDWARE PERFORMANCE COUNTERS
}

\author{
by \\ MARCO CHIAPPETTA
}

Submitted to the Graduate School of Engineering and Natural Sciences in partial fulfillment of the requirements for the degree of Master of Science

Sabancı University

August 2016 
(C) Marco Chiappetta 2016

All Rights Reserved 


\title{
REAL TIME DETECTION OF CACHE-BASED SIDE-CHANNEL ATTACKS USING HARDWARE PERFORMANCE COUNTERS
}

\author{
Marco Chiappetta \\ Computer Science and Engineering, Master's Thesis, 2016 \\ Thesis Advisor: Prof. Dr. Erkay Savaş \\ Thesis Co-Advisor: Assist. Prof. Dr. Cemal Yilmaz
}

\begin{abstract}
Cache-based side-channel attacks are increasingly exposing the weaknesses of many cryptographic libraries and tools by showing that, even though the algorithms might be considered strong, their implementations often lead to unexpected behaviors that can be exploited to obtain sensitive data, usually encryption keys. In this study we analyze three methods to detect cache-based side-channel attacks in real time, preventing or limiting the amount of leaked information. We focus our efforts on detecting three attacks on the well-known OpenSSL library: one that targets AES, one that targets RSA and one that targets ECDSA. The first method is based on monitoring the involved processes and assumes the victim process is known. By collecting and correlating the monitored data we find out whether there exists an attacker and pinpoint it. The second method uses anomaly detection techniques and assumes the benign processes and their behavior are known. By treating the attacker as a potential anomaly we understand whether an attack is in progress and which process is performing it. The last method is based on employing a neural network, a machine learning technique, to profile the attacker and to be able to recognize when a process that behaves suspiciously like the attacker is running. All the three of them can successfully detect an attack in about one fifth of the time required to complete it. We could not experience the presence of false positives in
\end{abstract}


our test environment and the overhead caused by the detection systems is negligible. We also analyze how the detection systems behave with a modified version of one of the spy processes. With some optimization we are confident these systems can be used in real world scenarios. 


\title{
DONANIM PERFORMANS SAYACLARI İLE ÖN-BELLEK KULLANILARAK YAPILAN YAN-KANAL SALDIRILARININ GERÇEK ZAMANLI OLARAK TESPITT EDİLMESI
}

\author{
Marco Chippetta \\ Bilgisayar Bilimi ve Mühendisliği \\ Yüksek lisans tez, 2016 \\ Tez Danışmanı: Prof.Dr. Erkay Savaş \\ Tez Eş Danışmanı: Assist. Prof.Dr. Cemal Yilmaz
}

\begin{abstract}
Özet
Ön-bellek kullanılarak gerçekleştirilen yan-kanal saldırıları giderek artan bir hızla birçok kriptografik yazılım kütüphanesinin zaaflarını ortaya çıkartmaktadır. Kullanılan kriptografik algoritmalar teorik olarak güvenilir olsa da, yazılım olarak gerçeklerken yapılan hatalar nedeniyle, algoritmanın çalışması sırasında beklenmeyen bilgiler dışarıya sızmakta ve bu yolla gizli anahtarlar elde edilebilmektedir. Bu çalışmada ön-bellek kullanılarak gerçekleştirilen yan-kanal saldırılarının gerçek zamanlı olarak tespit edilmesi, önlenmesi ya da sızan hassas/gizli bilgi miktarının en aza indirgenmesi icin önerdiğimiz üç farklı yöntem ele alınacaktır. Bu tez kapsamında yaygın olarak kullanılan OpenSSL yazılım kutuphanesinde gerçeklenmiş üç adet kriptografik algoritmaya karşı geliştirilen saldırılara odaklanacağı: blok şifreleme algoritması AES, RSA ve eliptik eğri elektronik imza (ECDSA) algoritmaları. Geliştirdiğimiz ilk yöntem saldırı yapılan kriptografik algoritmayı çalıştıran prosesi bildiğimizi kabul ederek sistemdeki ilgili prosesleri izlemeyi gerektirmektedir. İzledigimiz proseslerden elde edilen veriler arasında bir korelasyon bulmaya çalışarak, bir saldırının olup olmadı̆̆ı, var ise saldırgan prosesin tespit edilmesi hedeflenmektedir. İkinci yöntem, temel olarak ayrık değer bulma ya da anomali tespiti yaklaşımını esas almaktadır. Bu yöntemde saldırgan olmayan proseslerin ve bunların dinamik davranışlarının bilindiği varsayılmaktadır. Saldırgan prosesin dinamik davranışının anomali oluşturduğu kabulüyle, sistemde bir saldırgan prosesin olup olmadığı ve varsa hangisinin olduğunun bulunması amaçlanmaktadır. Önerilen son yöntemde ise, saldırgan prosesin davranışının makina öğrenmesi yöntemleri kullanılarak modellenmesi esas alınmaktadır. Önerilen üç yöntemde de, saldırının gerçekleşmesi için gereken za-
\end{abstract}


manın en fazla beşte biri kadar bir sürede, saldırı başarılı bir şekilde tespit edilebilmektedir. Yapılan deneylerde, hic pozitif yanlış durumu oluşmamıştır. Ayrıca saldırı tespit yöntemlerinin hız açısından sistem başarımındaki olumsuz etkisinin ihmal edilebilecek mertebelerde kaldığı gözlemlenmiştir. Saldırıyı gerçekleştiren prosesin farklı sürümleri kullanılarak saldırı tespit sisteminin başarımı da ölçülmüş̧ür. Geliştirilen saldırı tespit yöntem ve yazılımları, daha da iyileştirilerek gerçek dünya senaryolarında da kullanılabilecek niteliğe sahip hale getirilebilir. 


\section{Contents}

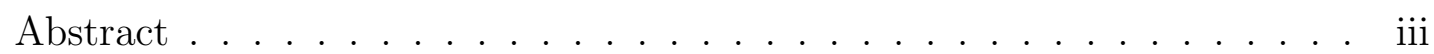

Özet . . . . . . . . . . . . . . . . . . . . v

1 Introduction 1

2 Background $\quad 4$

2.1 Cache-based side-channel attacks ............... . . 4

2.2 Attacking RSA . . . . . . . . . . . . . . 7

2.3 Attacking AES . . . . . . . . . . . . . . . . 8

2.4 Attacking ECDSA . . . . . . . . . . . . . . . 10

2.5 Hardware Performance Counters . . . . . . . . . . . . . . . . . . . 14

2.6 Anomaly detection . . . . . . . . . . . . . . . . . 16

2.7 Supervised Learning and

Neural Networks . . . . . . . . . . . . . . . . 17

3 Detecting a spy process $\quad 20$

3.1 Correlation based approach . . . . . . . . . . . . . 21

3.2 Anomaly detection based approach . . . . . . . . . . . 26

3.3 Based on supervised learning . . . . . . . . . . . . . . 27

4 Experiments and results $\quad 29$

4.1 Overview . . . . . . . . . . . . . . . . . . . . 29

4.2 Detecting AES spy process . . . . . . . . . . . . . . . 32

4.3 Detecting ECDSA spy process . . . . . . . . . . . . 34

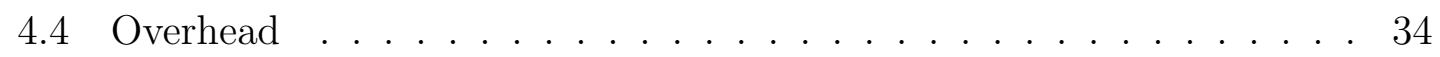

$\begin{array}{lll}5 & \text { A smarter spy process } & 36\end{array}$ 
6 Discussion

7 Conclusion and future work 


\section{List of Figures}

2.1 Main loop of the Montgomery ladder implementation in OpenSSL . . 12

2.2 Sample output of the perf-stat utility . . . . . . . . . . . . 15

2.3 Representation of a simple neural network . . . . . . . . . . . . 18

3.1 Total L3 cache accesses of spy and victim of the attack to ECDSA. The similarities are visible between samples 200 and 550 when the Montgomery ladder loop is executed. . . . . . . . . . . . . . 22

3.2 Total L3 cache accesses of the spy and the victim of the attack to AES. 22

3.3 Total L3 cache accesses of the Apache webserver serving a 211 B HTML file 1000 times with 100 concurrent clients and the victim of the attack to ECDSA. The difference in cache accesses over time is so high that the line corresponding to the victim process is barely visible. 24

3.4 Total L3 cache accesses of the Apache webserver serving a 1 MB JPG file 1000 times with 100 concurrent clients and the victim of the attack to ECDSA. . . . . . . . . . . . . . . . 25

3.5 Total L3 cache accesses of the Apache webserver serving the output of a PHP script calling php_info 1000 times with 100 concurrent clients and the victim of the attack to ECDSA. The difference in cache accesses over time is so high that the line corresponding to the victim process is barely visible. . . . . . . . . . . . . . . . 25

3.6 In this example different circles representing distinct values of epsilon, the threshold for the density estimation function, visually show how anomalies are flagged according to the value picked. . . . . . . . . . 27 
4.1 Relationship between the number of samples collected during an attack to AES and the confidence of the prediction based on correlation. Even though the relationship is not linear (since the confidence is influenced by noise caused by other processes, scheduling policies etc.) the general trend is that the higher the number of samples the higher the confidence. . . . . . . . . . . . . . . . . 33

5.1 Relationship between the total LLC accesses of the AES victim process and the modified version of the spy process. . . . . . . . . 37 


\section{List of Tables}

4.1 Benchmarks of the detection method based on correlation . . . . . . . 30

4.2 Benchmarks of various operations . . . . . . . . . . . . . . 31

4.3 Benchmarks of the detection methods based on machine learning tech-

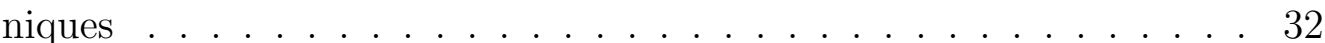

5.1 Confidence values and execution time for the three variants of the modified version of the AES spy process. Each variant sets a different value for the maximum number of addresses that are probed for each iteration. . . . . . . . . . . . . . . . . . 38 


\section{Chapter 1}

\section{Introduction}

Side-channel attacks are a particular class of attacks, usually targeting cryptographic algorithms, which do not exploit a flaw in the design of the algorithms themselves but rather in their implementation.

Cache-based side-channel attacks represent a subset whose purpose is to retrieve sensitive information from a system just by exploiting the shared cache memory in modern CPUs [2]. Moreover such attacks can be conducted between virtually isolated environments such as virtual machines or Linux containers as shown in [11], [12] and [13].

As described in the next chapter, a class of cache-based attacks rely on the presence of an assembly instruction to partially or fully manipulate the state of the shared cache (in the case of Intel CPUs the instruction is CLFLUSH) and the presence of a feature, such as Kernel Same-page Merging (KSM) [3] or Transparent Page Sharing (TPS) [4], which allows processes to share identical pages in memory.

To prevent such attacks between processes or virtual machines, we would either need to switch to a CPU architecture that prevents the usage of the aforementioned instruction or to disable any memory optimization feature. In the first case, it would be necessary to recompile any incompatible program for the new architecture (e.g. ARM) while in the second case there would be a loss of performance given by the fact that processes would be unable to share identical pages, therefore increasing memory consumption.

With regard to virtual machines, another problem is co-location. That is, to carry out the attack it is necessary that the attacker's virtual machine and the vic- 
tim's virtual machine run on the same physical hardware, therefore sharing the main memory and the cache. Until Amazon upgraded their platform, implementing the proper countermeasures, such problem had been partially solved by Ristenpart et al. [5] who were able to colocate two virtual machines on the Amazon EC2 cloud computing service with a probability of $40 \%$. More recently Inci et al. [6] showed a new, more accurate, approach to achieve and detect co-location, again on Amazon EC2, by monitoring the last level cache. The link between co-location and exploitation is established in [13], where Irazoqui et al. managed to detect the version of popular cryptographic libraries, such as OpenSSL and PolarSSL, in a cross-VM scenario using the Kernel-based Virtual Machine (KVM) [38] supervisor, simplifying the process of detecting whether a target is vulnerable to specific cache-based attacks.

The first practical implementation of a cache-based attack was presented by Tsunoo et al. in [7] and targeted the DES algorithm. In [8] Osvik et al. devised two techniques (EVICT+PRIME and the more efficient PRIME+PROBE) to attack AES by evicting everything in the cache and measuring the time for an encryption. More recent cache attacks include [9], by Yarom and Falkner, that uses the FLUSH+RELOAD technique to retrieve the private exponent used in GNU Privacy Guard (GPG)'s implementation of RSA, [10] by Yarom and Benger where the same technique is used against the ECDSA implementation in OpenSSL and [11], by Irazoqui et al., and [12], by Gulmezoglu et al., where FLUSH+RELOAD is used to detect the key used in the last round of an AES encryption.

The problem we address is to detect such attacks in time, before they are complete, to be able to take the proper countermeasures, i.e. to kill the suspicious process, in a same-OS scenario, or relocate the virtual machine, in a cross-VM scenario. A similar attempt at detecting this kind of attacks is [16].

In this study we present three methods, of which two are based on machine learning techniques, that can be combined or used separately to detect cache-based sidechannel attacks at runtime, with a particular focus on those using the FLUSH+RELOAD technique [9]. Our methods do not require any modification to the operating system and run as normal user-level processes. The only requirement is the availability of hardware performance counters, quite common on most modern CPUs [25]. 
The organization is as follows: in Chapter 2 we present necessary background information on cache-based side-channel attacks and hardware performance counters followed by an analysis of three attacks against RSA, AES and ECDSA. In Chapter 3 we describe our methods and their advantages and shortcomings. In Chapter 4 we show our results and how it is possible to detect an attack in time to take the proper countermeasures; we also analyze the overhead caused by our detection system. Chapter 5 presents an improved version of one of the attacks that is able to deceive the first (and simplest) detection method while still being able to complete an attack, although in more time. We believe this might trigger interest in further research on how to deceive, and therefore improve, detection systems for these kinds of attacks. Chapters 6 and 7 present a discussion about our results, and their implications, and the feasibility of employing such detection systems in real world scenarios. 


\section{Chapter 2}

\section{Background}

In this chapter we introduce background information necessary to better understand the attacks themselves and the techniques used to detect them.

\subsection{Cache-based side-channel attacks}

Numerous attacks based on shared hardware and software resources have been carried out in the past. Recently those based on CPU's cache memory turned out to be very effective, easy to implement and fast. This study focuses on a particular class of cache-based side-channel attacks that utilize a technique named FLUSH+RELOAD [9].

Modern CPUs utilize one or more layers of cache [18] to speed up frequent operations by decreasing the average access time of content stored in the main memory. Most Intel CPUs have 3 levels of cache with increasing access time: L1 (usually split into L1i for instructions and L1d for data), L2 and L3. The last two levels are shared among the CPU cores. Furthermore processes can arbitrarily flush specific memory addresses from the CPU's cache by using a specific assembly instruction. On most modern Intel processors (mainly Core i3, i5, i7 and Xeon) such instruction exists under the assembly mnemonic CLFLUSH [20]. The shared cache and the unregulated access to this kind of instructions are what makes possible most of the attacks analyzed in this study.

These cache memories operate on units of $64 \mathrm{~B}$ called lines. That is, let $P_{1}$ and $P_{2}$ be two processes running on separate cores. If $P_{1}$ accesses address $a_{1}$ what will be loaded into the cache is not just the content of $a_{1}$ but a block of $64 \mathrm{~B}$ (i.e. a 
line) containing $a_{1}$ (the block's start and end addresses depend on the main memory alignment). Therefore if $P_{2}$ calls CLFLUSH on $a_{1}$ what will be flushed is not just the content of $a_{1}$ but the entire line.

The entities usually involved in attacks based on FLUSH+RELOAD are indeed two processes: a victim and a spy. The victim performs some kind of cryptographic operation (i.e. encryption, decryption or signature) where some secret data, likely a key, are being used while the spy attempts to capture such data by analyzing the victim's behavior.

The success of the attack mainly depends on three factors: the ability of the spy to synchronize with the victim (that is, to start the attack as soon as the cryptographic operation starts), the presence of a user-level instruction to evict a specific area of the CPU's cache (such as CLFLUSH) and, in the case of virtual machines, the presence of mechanisms such as Transparent Page Sharing (TPS) [4] or Kernel Same-page Merging (KSM) [3].

KSM was implemented for the first time in Linux 2.6.32 as a technique to augment memory density and it is enabled by default. It allows processes to transparently share identical pages by mapping addresses which belong to different virtual address spaces to the same physical address. Two downsides of KSM are the high CPU load needed to regularly run the merging process [15] and the fact that it makes attacks such as FLUSH+RELOAD feasible.

TPS is, instead, a proprietary technology of VMWare whose purpose is to make virtual machines share identical pages with the hypervisor taking care of searching and merging them. The feature is enabled by default in both their cloud and desktop solutions until the latest version (6.0 at the time of writing) [17] where it has to be manually enabled because of security concerns [19].

Since two merged pages are mapped to the same physical address, in the main memory, different processes that try to retrieve a shared page cause the MMU (Memory Management Unit) [27] to access the same physical address. Furthermore the cache, being physically indexed, is mapped onto the same address space and content that is evicted from it will be evicted for all processes that share it in the main memory.

Calling CLFLUSH with a single address causes the whole cache line, which includes 
the content from that address, to be evicted. Furthermore, on Intel CPUs, cache levels form an inclusive hierarchy: the L3 cache includes the L2 cache content and the L2 cache includes the L1 cache content. For this reason evicting a line from the LLC (L3) propagates the eviction to the lower levels as well. The algorithm roughly works as described in Example 1.

Example 1. Algorithm for a generic FLUSH+RELOAD attack.

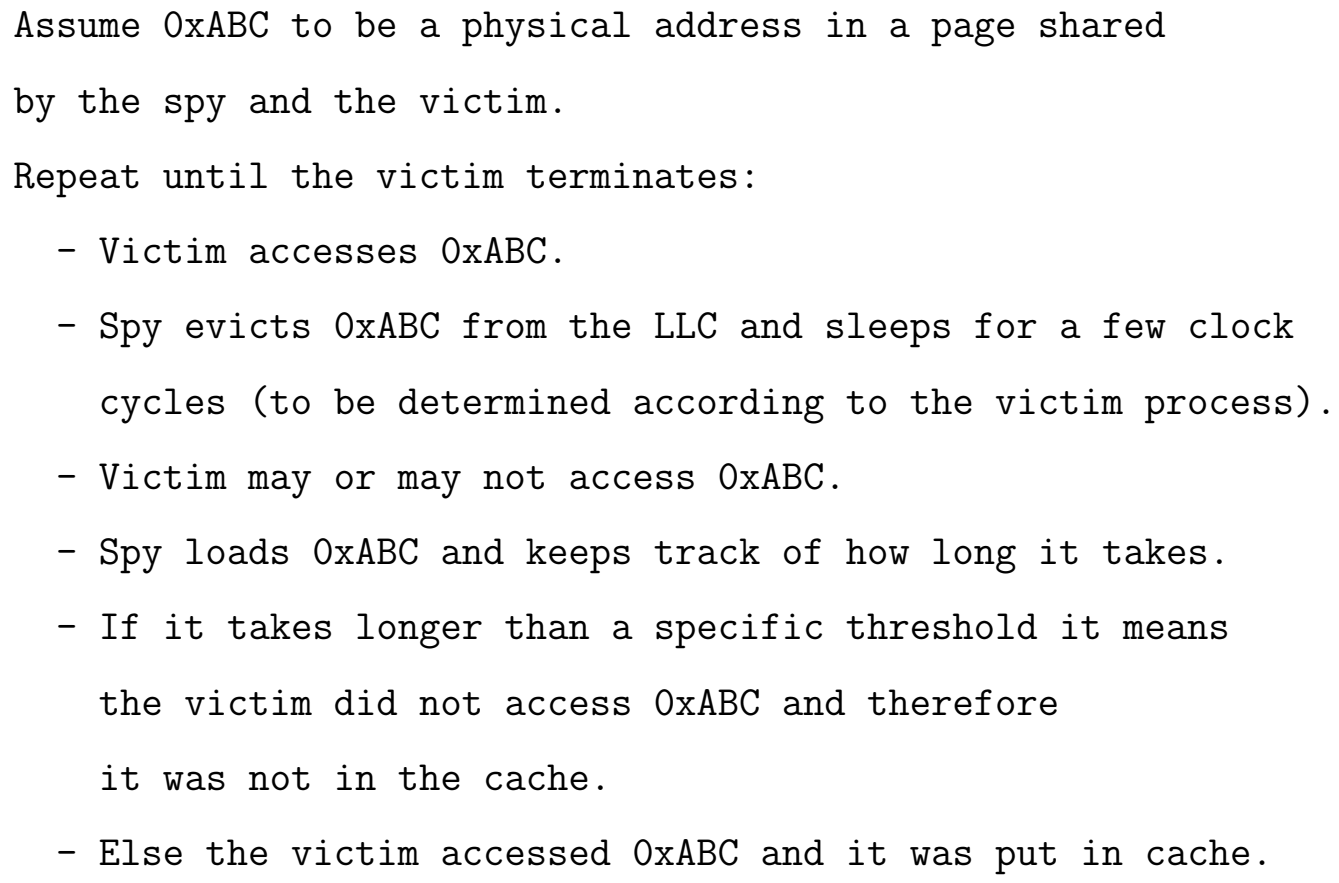

The original FLUSH+RELOAD attack [9], by Yarom et al., focused on guessing which instructions are being executed by the victim. In fact, by knowing which instructions are or are not executed while performing a cryptographic operation it is often possible to retrieve information that can be used to reconstruct the secret used during the process, such as encryption keys. In particular the first attack described in the paper is used to determine the bits of the key used in GPG when performing a decryption with RSA, thanks to a vulnerable implementation of the square multiply algorithm.

To address the concern that this attack would only work on non-constant-time implementations (that is, implementations whose execution time highly depends on the input, especially sensitive input such as encryption keys, that determine which instructions are executed), a second version of the attack was released, this time aimed at breaking the supposedly robust implementation of the elliptic curve point 
scalar multiplication algorithm based on the Montgomery ladder used in OpenSSL's ECDSA [10].

A variant of such technique [11], i.e. the third type of attack, by Irazoqui et al. was able to retrieve all the AES last round's key bits by observing a few seconds to a minute worth of encryptions or decryptions, although the amount of time required to complete an attack makes it more prone to be detected as demonstrated by our experiments in Chapter 4.

In our work we aimed at detecting the second and third types of attack, the first being a simpler version of the second.

\section{$2.2 \quad$ Attacking RSA}

While performing a signature or a decryption with RSA there is the need to compute $m^{d} \bmod n$ where $m$ is the plaintext, $d$ is the private exponent and $n$ is the product of two large prime integers. One algorithm to perform such computation is square multiply, also known as binary exponentiation, described in Algorithm 1. Here the function $\operatorname{bin}(d)$ returns the individual bits of the private exponent as an array of integers.

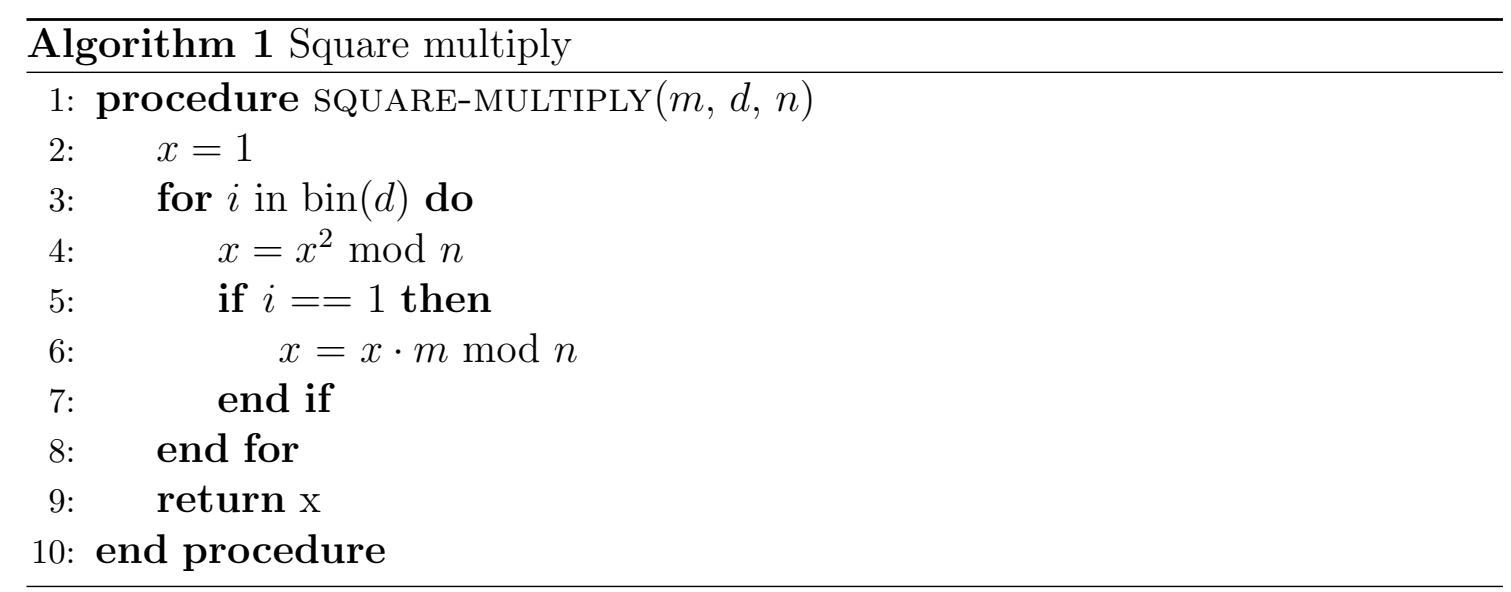

Given the non-constant time nature of the algorithm its implementations are vulnerable to different kinds of side-channel attacks, including those based on timing and power analysis [21]. In particular the operations performed according to the value of each key bit leak valuable information that can be used to reconstruct the key. 


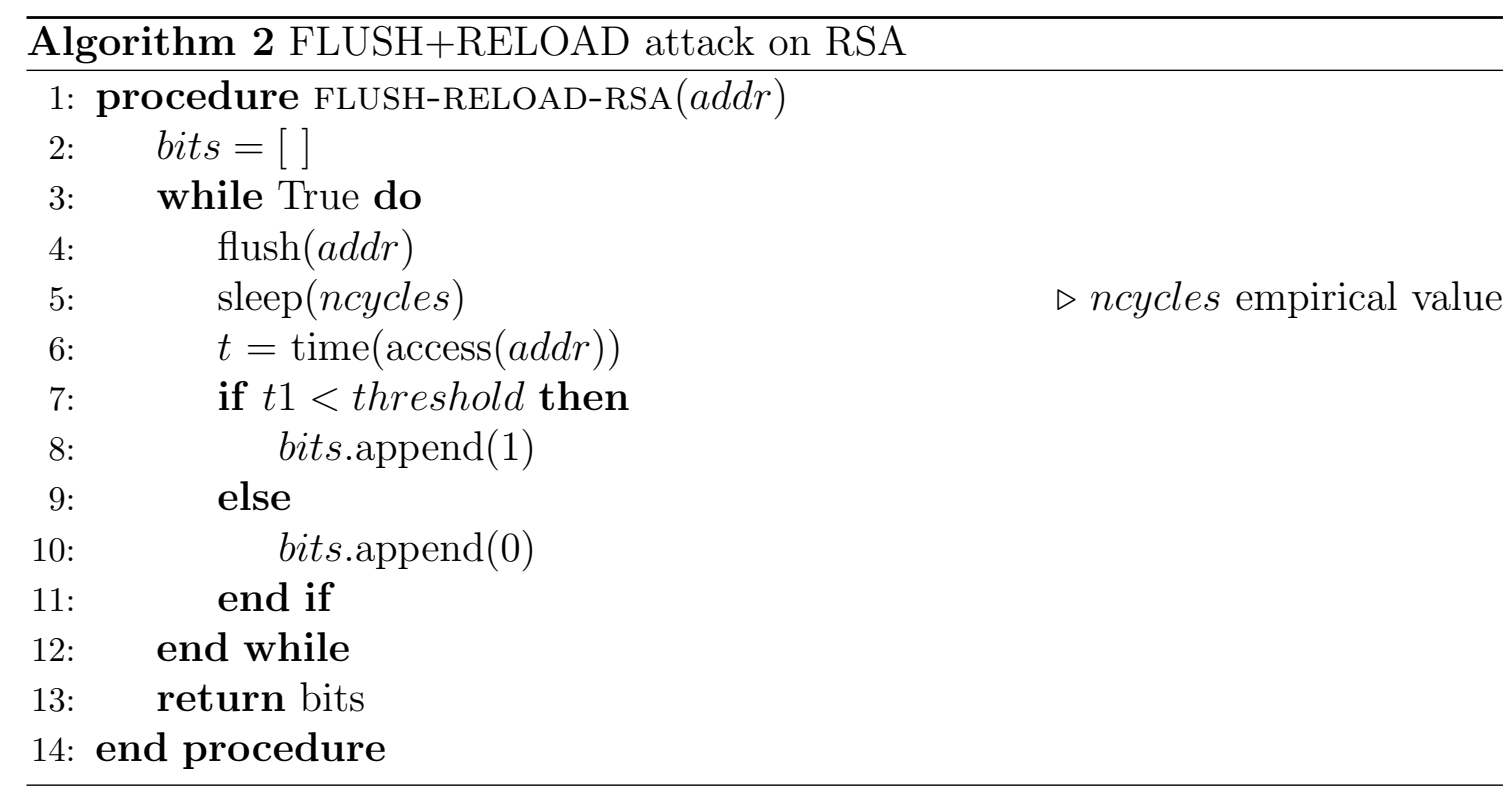

In [9] Yarom et al. exploited the implementation of the square multiply algorithm inside GPG. By reverse engineering the OpenSSL binary it is possible to retrieve the memory address of line 6, from Algorithm 1, in the GPG binary and use it to carry the attack described in Algorithm 2.

The attack briefly works as follows: the spy starts executing a loop in which it first flushes the targeted address, in line 4, then waits an empirically determined number of cycles before reloading the address, in line 6. When the spy reloads the address there exist two possibilities corresponding to the two branches of the conditional at line 7: if the victim accessed its content the loading time will be lower than a predefined threshold, which means the bit is likely 1 , otherwise it will be higher, which means the bit is likely 0 .

In [9] the authors reported that, on average, the percentage of the private exponent's bits that can be recovered is $96.7 \%$ with a worst case of $90 \%$, in a cross-VM scenario, and $98.7 \%$ with a worst case of $95 \%$ on the same operating system.

\subsection{Attacking AES}

It is possible to perform a known-ciphertext attack on common implementations of AES (such as the one used in OpenSSL). What makes this kind of attack feasible is the use of lookup tables (often named T-tables or T-boxes), originally proposed in [1], to optimize the operations needed to compute a single AES round (i.e. KeyAddition, 
SubBytes, ShiftRows, MixColumns) into some table lookups and XOR operations.

These tables, being used frequently, are usually loaded in the CPU's cache once they are accessed. With some reverse engineering it is possible to find the memory locations where the tables will be stored at runtime. Once the locations are known the attacker can understand whether a given table was accessed by loading its corresponding memory address and looking at the loading time. For each round the attacker flushes the tables from the cache, waits until the victim uses the tables and then understands which table was used by employing the FLUSH+RELOAD technique.

Irazoqui et al. devised an algorithm to fully recover the scheduled key used in the last round of AES in a matter of seconds to minutes [11]. As already mentioned, their algorithm is a variant of the more generic FLUSH+RELOAD that focuses on guessing which values of the AES lookup tables were accessed and uses this information to reconstruct the round key. In a byte-oriented implementation of AES the i-th ciphertext byte in the last round is produced as follows:

$$
C_{i}=T\left[S_{i}\right] \text { XOR } K_{i}
$$

where $T$ is the lookup table, $S_{i}$ is the i-th byte of the current state, used as an index for $T$, and $K_{i}$ is the i-th byte of the round key.

Let $T$ be the lookup table and let us assume that the monitored cache line holds the first $n$ table entries. It is not possible to understand precisely which of the $n$ entries is used but we know that, in general, whenever one of the entries is accessed, $T$ is accessed. Every time this happens we store the corresponding ciphertext byte and create a set of all 16 possible values for $S_{i}$ and $K_{i}$ for that ciphertext byte. By repeating the steps for multiple ciphertext bytes, we should see a common value in each set which is likely the correct key's byte.

For further details refer to [11], Section 5.1. 


\subsection{Attacking ECDSA}

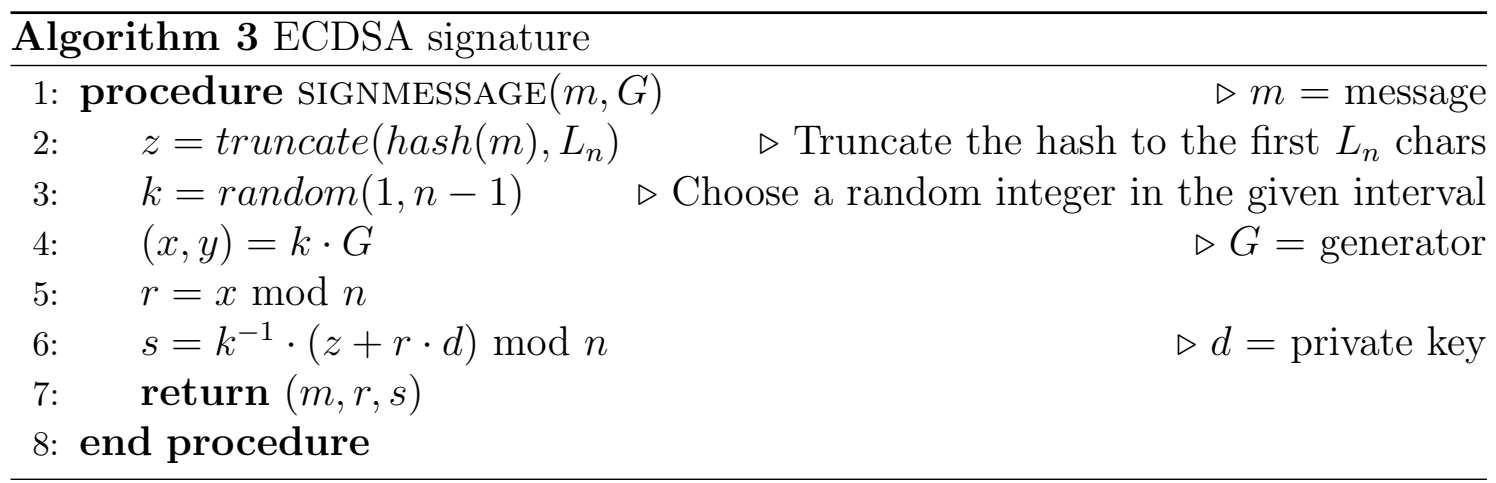

A message signed with ECDSA consists of a triple $(m, r, s)$ where $m$ is the message and $r$ and $s$ are computed as in Algorithm 3. We assume an elliptic curve group of order $n$ and that $G$ is a generator of such group. Specifically the curve used in the attack is sect571r1 whose parameters are described in [22].

The ephemeral key $k$ used in the signature algorithm can be exploited to retrieve the private key $d$ since $d=(s k-z) r^{-1}$ and $s, z$ and $r$ are known (see Algorithm 3).

Attacking an implementation of the signature algorithm means, indeed, attacking the step where the elliptic curve point $(x, y)$ is computed, as shown in Step 4 of Algorithm 3. In fact the implementation of the point multiplication algorithm used for the computation can lead to some data leakage that provides information for an attacker to reconstruct the ephemeral key.

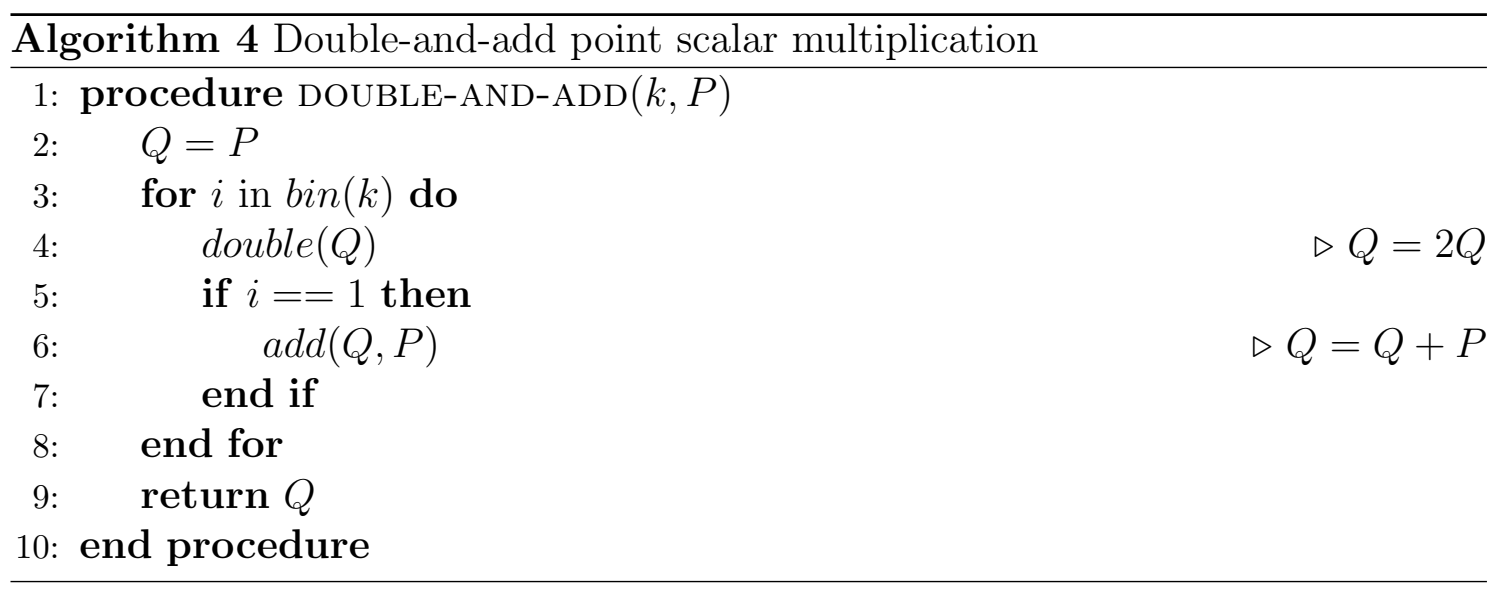

A simple implementation of the point multiplication algorithm, called doubleand-add, is provided in Algorithm 4. Such implementation could be exploited with the same process shown in the previous chapter. 
In fact it can be noticed that by using a simple attack based on FLUSH+RELOAD we can guess when a bit is 0 or 1 by monitoring the cache line corresponding to the function called at Step 6 in Algorithm 4. Whenever the bit is 1 the line will be loaded in cache by the victim and the loading time in the spy will be shorter otherwise it is fair to assume the bit is 0 .

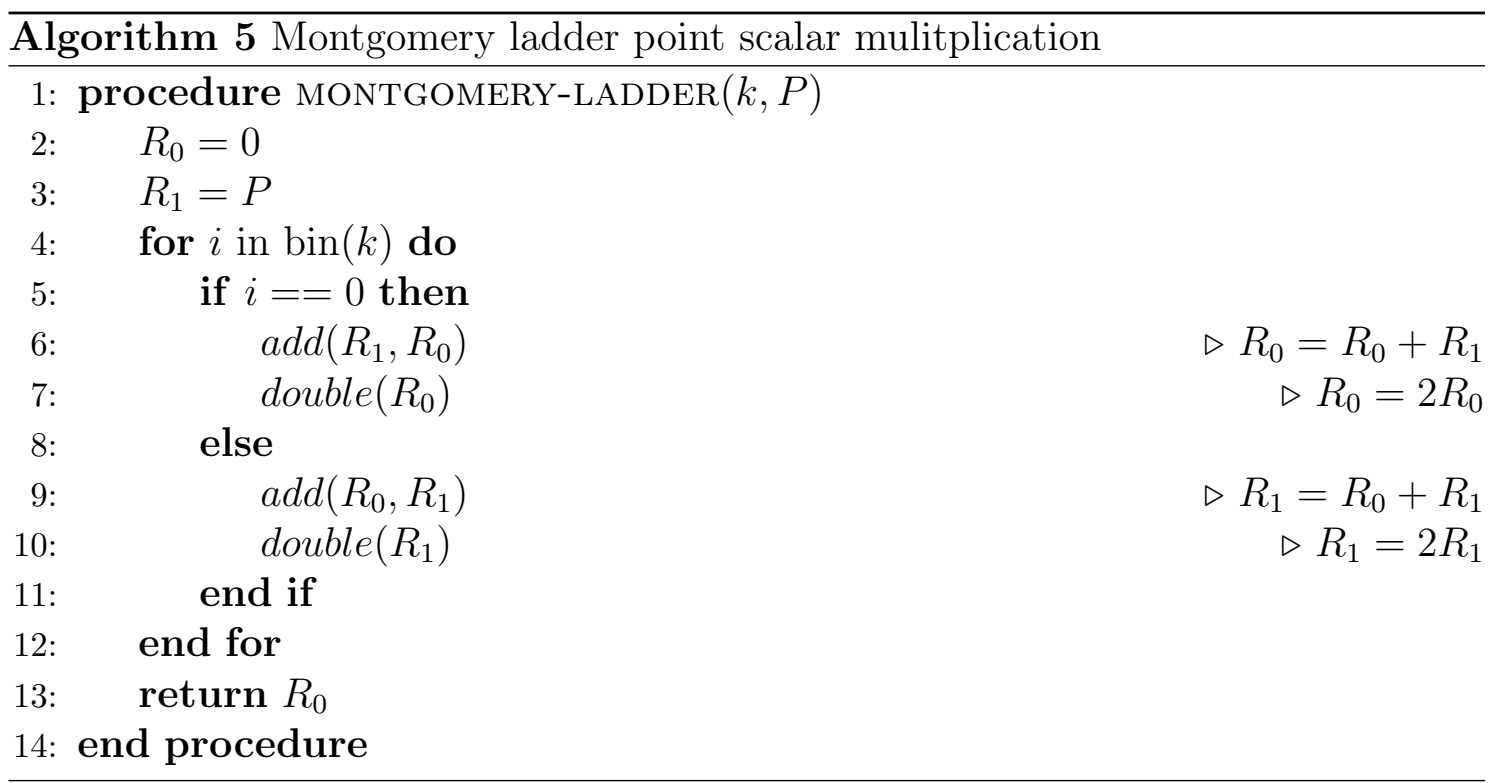

To avoid this kind of attacks, OpenSSL uses a different implementation based on the Montgomery ladder [23] described in Algorithm 5. The Montgomery ladder relies on the same functions being called regardless of whether the bit is clear or set. The only change between the two cases is in the order of the arguments passed to the functions. 


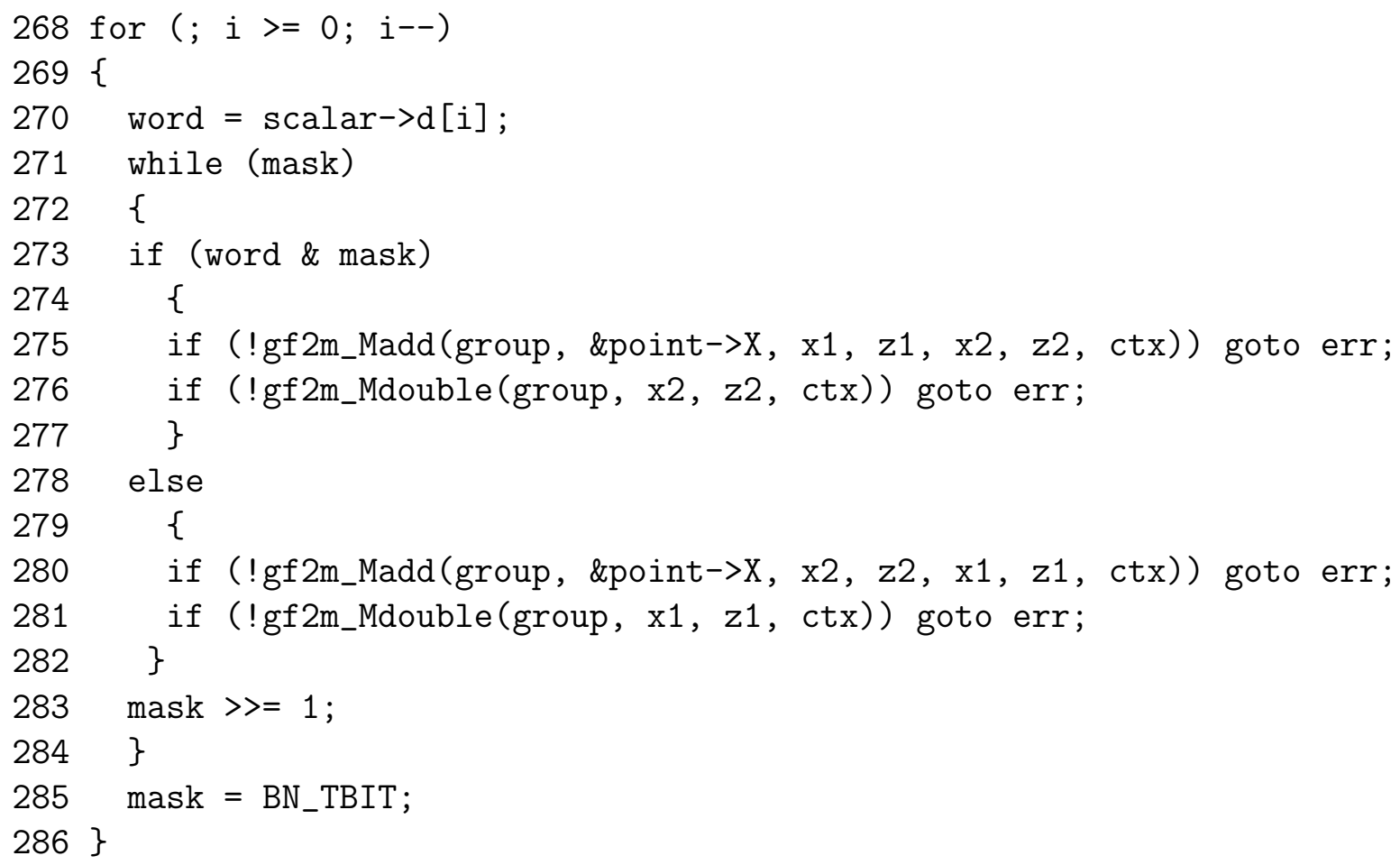

Figure 2.1: Main loop of the Montgomery ladder implementation in OpenSSL

OpenSSL's implementation was broken by Yarom et al. [10] proving that FLUSH+RELOAD can be used even when the algorithm is supposed to resist against timing attacks. The target of the attack is the code contained in function ec_GF2m_montgomery_point_multiply, a sample of which is shown in Figure 2.1.

To perform the point scalar multiplication using the Montgomery ladder the scalar $k$ is read bit by bit in a loop. According to the value of each bit a different conditional branch is taken and the functions to add and double the point, gf $2 \mathrm{~m} \_$Madd and gf2m_Mdouble, are called with the arguments in a different order. The principle behind this design is that since the same functions are called regardless of the state of the current bit, an attack based on timing would fail.

With some reverse engineering on the OpenSSL binary it is possible to retrieve the memory addresses of the lines of interest: 275, 276, 280 and 281. With spatial prefetching [24] the content of the cache is optimized by copying not only the line that contains the addresses being accessed but also a limited number of adjacent lines, therefore it is necessary to probe addresses that are as distant as possible from each other in memory (and consequently in the cache) to avoid false positives caused by this feature. 


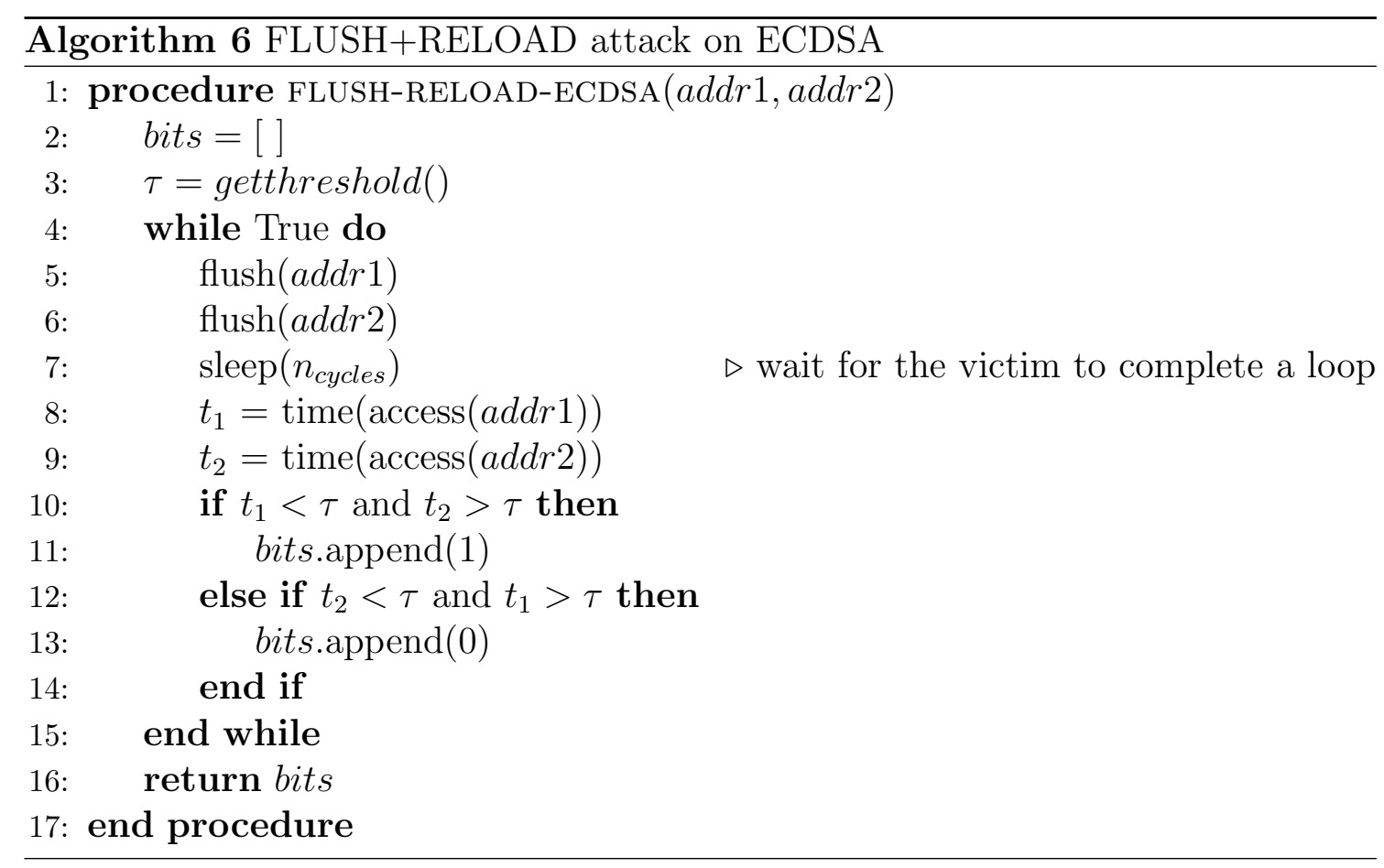

Specifically lines 275 and 281 of Figure 2.1, whose memory addresses are passed as arguments addr1 and addr2 in Algorithm 6, are suitable for the attack since they lie at the very beginning and the very end of the main conditional branch of line 273. The attack proceeds by flushing and reloading these addresses to understand which ones were accessed.

Assuming the spy starts executing the loop in Algorithm 6 and at the same time the victim starts the main loop in Algorithm 5 the two processes are perfectly synchronized and the attack has the highest likelihood of success.

It is necessary, for the spy, to sleep for a certain amount of CPU cycles $\left(n_{\text {cycles }}\right.$ in Algorithm 6) equal to the average number of cycles needed for the victim to complete a loop. The actual time is not always constant but depends on how the processes are scheduled.

In line 10 of Algorithm 6 other than just checking whether $t_{1}<\tau$ we also check whether $t_{2}>\tau$. That is, we make sure that $a d d r 1$ was not loaded in memory because of spatial prefetching. Because of spatial prefetching if two addresses belong to the same set of lines loaded from the main memory it is impossible to understand which one was intentionally loaded by a process and which one was retrieved because of this feature. In this case we want to make sure that addr1 was not loaded in the cache just because addr2 was (and viceversa in line 12). 
Another issue arises when the spy has to terminate. If it terminates too soon it will miss some of the last bits so the best course of action would be to keep executing the loop up to a point when a certain number of bits are equal to 0 (i.e. both $t_{1}$ and $t_{2}$ are above the threshold) indicating those addresses are not being accessed anymore.

At the end of the attack some (or all) of the bits of the ephemeral key are recovered and it is possible to reconstruct the private key. In the worst case the attack is known to miss 34 bits but the actual value of the scalar $k$ can be restored, by using the baby step giant step algorithm, in less than one second of computation and using just $10 \mathrm{MB}$ of memory [10].

\subsection{Hardware Performance Counters}

Modern microprocessors are equipped with special purpose registers used to store data about a wide range of CPU related events: clock cycles, cache hits, cache misses, branch misses etc. Such registers, called Hardware Performance Counters (in short HPCs), are commonly used to profile the behavior of a program and understand what to optimize in order to increase its performance [25]. In this study we describe an alternative usage of such feature that allows us to collect predictive data about one or more processes with little overhead.

Similar alternative usages are described in [30] where the timing function of a particular time based cache attack is replaced with data coming from HPCs, in [31] where malwares are detected by constructing a dynamic signature of the processes involved and in [32] which briefly mentions how it would be possible to mitigate the effects of some classes of cache-based side-channel attacks through the use of HPCs.

The Linux kernel, assuming the target CPU supports them, provides an interactive interface to the HPCs via a command-line tool named perf [26]. The tool allows to collect, visualize, filter and aggregate data gathered through the HPCs on a system-wide, process or even thread basis.

The most interesting sub-command, for the purposes of our experiments, is perf-stat. Using this utility it is possible to specify which events to monitor, a target process or thread, the output format and the interval of time between 
two consecutive reports. An example report from perf-stat, while monitoring the execution of the tool make, is shown in Figure 2.2.

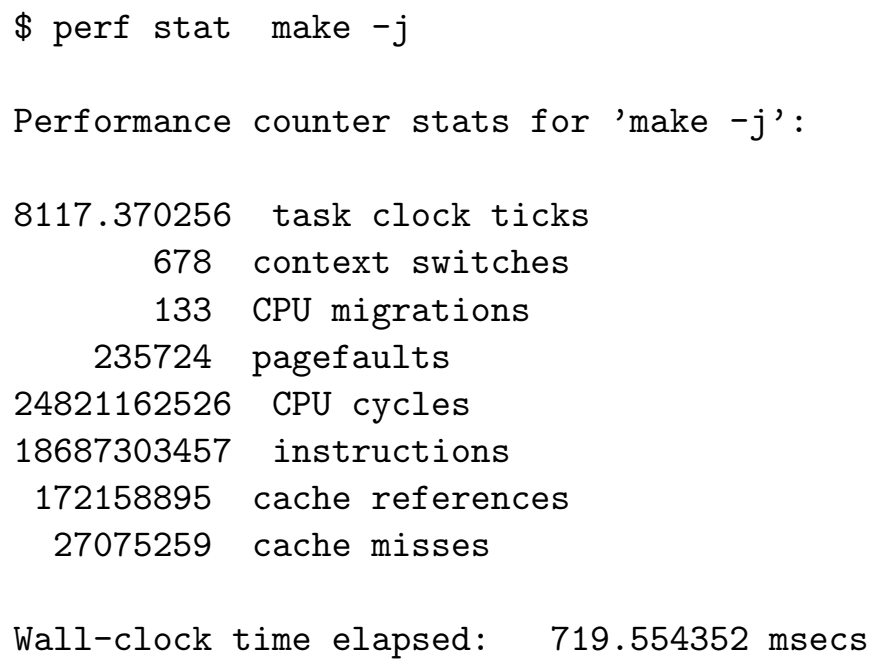

Figure 2.2: Sample output of the perf-stat utility

An important shortcoming of perf-stat is its limited resolution; perf-stat gives the opportunity to sample HPCs multiple times in a second but the minimum interval between two consecutive samples is $100 \mathrm{~ms}$, much higher than the time necessary to complete some of the attacks, as shown in Chapter 4.

For this reason we developed a custom utility, called quickhpc [40] that offers a subset of the features of perf-stat but with some improvements. The tool can be run as a normal user level process and requires the privileges used by the process that should be monitored (e.g. if the process to monitor was run as root quickhpc has to be run as root as well). When running quickhpc the required arguments are the PID of the process to monitor and the list of events to be monitored. Optionally, it is possible to specify the maximum number of samples and the interval in microseconds between two samples.

The library used for probing HPCs is PAPI (Performance Application Programming Interface) [28]. The main reason quickhpc uses PAPI is its high resolution. After a thorough optimization quickhpc reaches a maximum resolution (i.e. the time between two samples) of 3 microseconds, more than 30000 times faster than perf-stat.

It is worth noting that the resolution of quickhpc is not fixed but is influenced by 
the workload on the system, the scheduling policy, the behavior process monitored and so on. Also the number of collected samples, for the same process, may vary each time since it is not possible to start the monitored process and quickhpc at exactly the same instant; it all depends on the scheduling policy set in the operating system.

\subsection{Anomaly detection}

Anomaly detection is used to find outliers, or anomalies, in an unlabeled dataset. Some examples of real world problems, where anomaly detection plays an important role, are detection of faulty products in factories and detection of fraudulent transactions.

The assumption is that there exists a set of features, for each instance, or sample, in the dataset, that can let us determine whether the instance belongs to a specific model (e.g. "legitimate transactions") or not. Let us indicate with $x_{j}^{(i)}$ the $\mathrm{j}$-th feature of the i-th instance in the dataset.

The aim is to retrieve a good number of samples considered "good" and find a probabilistic model that fits them. A usual assumption is that each feature $x_{j}$ fits a Gaussian distribution with mean and variance relative to that feature's values across all samples.

Therefore finding a model for feature $x_{j}$ means finding $\mu_{j}$ and $\sigma_{j}^{2}$ such that $x_{j} \sim \mathcal{N}\left(\mu_{j}, \sigma_{j}^{2}\right)$. Once these values are found the model can be tested by computing the distribution's density function for a new sample (i.e. probability that the given value $x$ belongs to a Gaussian distribution with the given mean and variance):

$$
p\left(x_{j} ; \mu, \sigma^{2}\right)=\frac{1}{\sqrt{2 \pi} \sigma} \exp \left(-\frac{\left(x_{j}-\mu\right)^{2}}{2 \sigma^{2}}\right)
$$

The value returned by this function has to be compared to a threshold which can be determined, in turn, by testing the model on a dataset that contains known anomalies. This allows to find a threshold that clearly separates the anomalies from the normal samples, very similarly the purpose of a classifier in the domain of supervised learning explained in the next chapter. For each new sample this probability is computed for each feature. The total probability is computed as follows: 


$$
p\left(x^{(i)}\right)=p\left(x_{1}^{(i)} ; \mu_{1}, \sigma_{1}^{2}\right) p\left(x_{2}^{(i)} ; \mu_{2}, \sigma_{2}^{2}\right) \ldots p\left(x_{n}^{(i)} ; \mu_{n}, \sigma_{n}^{2}\right)
$$

In our experiments we tried to fit a model for each kind of spy process implementation and considered all other (benign) processes as anomalies. The reason for not acting in the opposite way is that it is usually impossible to fit a model for all kinds of processes running on a system.

\section{7 $\quad$ Supervised Learning and Neural Networks}

The purpose of supervised learning is to construct models (classifiers) that are able to make predictions based on labeled data that were previously collected. Unlike unsupervised learning (where the purpose is to find patterns in non-labeled data) a datum, or sample, fed to a classifier, for the training phase, contains a vector of values named features (or independent variables) and a label whose value is a function of them (dependent variable).

The classifier is then trained by using a relatively large number of samples, aggregated in what is called the training set. Upon completion of each training phase a cross-validation and a test set, consisting of data not present in the training set, can be used to assess the effectiveness of the classifier.

Once the training phase is complete it is possible to feed the classifier with a single vector of features, omitting a label, which should be able to assign the label of the correct class to the given vector of features (the confidence of the prediction being dependent on a wide range of parameters). The hardest task in supervised learning is to find features that well characterize a certain class.

Although the principles behind supervised learning are similar to the ones behind anomaly detection there are a few key differences. In anomaly detection the classes are naturally skewed since the number of positives, i.e. anomalies, and negatives differ by several orders of magnitude (e.g. 1000 normal samples and 10 anomalies) where in supervised learning the more balance, between samples of different classes, the better; furthermore an anomaly detection mechanism does not distinguish between two or more classes but is only able to tell whether a sample belongs to the 
main class or not. In this study we explored both options, taking into account their advantages and disadvantages.

Neural networks are intended to represent a set of classifiers inspired by how neurons collaborate in a brain to accomplish some tasks, hence the name. A commonly used model is the feedforward network.

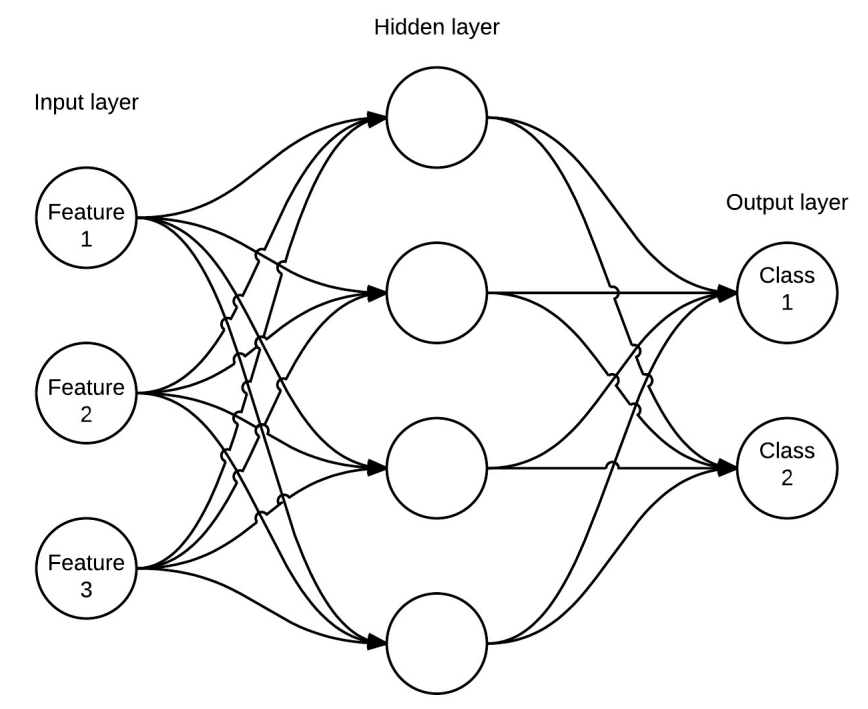

Figure 2.3: Representation of a simple neural network

As shown in Figure 2.3 such a network is formed by multiple layers. Each layer contains a certain number of neurons (or units), that hold a numerical value, called weight, and neurons of adjacent layers are connected to each other.

The vector of features is distributed among the neurons in the input layer and, after executing a feedforward propagation, the neurons in the output layer contain a vector of values whose maximum determines the class, i.e. its index in the vector, that the features supposedly belong to.

To train a neural network the feedforward propagation is followed by a backpropagation [35] step. In this step an error on the prediction is computed. Such error is then utilized, through the gradient descent algorithm, to adjust the weights of the neurons in the hidden layers to improve the accuracy of future predictions.

A common problem with supervised learning is overfitting. That is, the neural network precisely fits the training set but performs poorly on new, unlabeled samples. Such problem is partially solved by applying a technique named regularization [36] during the training phase. 
The metric we used to assess how well both the neural network and the anomaly detection system performed is their F-score [34]. This metric is more reliable than merely measuring accuracy (i.e. right predictions over all predictions) since it is not influenced by datasets where some classes contain a larger number of samples than others, called skewed classes.

Thanks to neural networks we are able to devise a more sophisticated mechanism for detecting a spy process, compared to correlation and anomaly detection, that decreases the chances of incurring in false positives and serves as an initial attempt to detect spy processes that employ strategies to avoid being uncovered. 


\section{Chapter 3}

\section{Detecting a spy process}

In this chapter we present three methods for detecting spy processes that exploit the FLUSH+RELOAD technique to perform cache-based side-channel attacks. All detection methods can successfully detect a spy before the attack is complete, therefore allowing to take appropriate countermeasures in time to prevent a leakage.

The first method is based on finding a correlation between the victim and the spy by analyzing the data collected by quickhpc. The intuition is that, in all the attacks we analyzed, both the spy and the victim processes, so far as the memory accesses are concerned, behave approximately the same way: the main operations are performed in a loop where specific, fixed memory addresses are accessed. Since the number of memory addresses accessed at each iteration is the same and both attacks work by flushing and reloading data from the L3 cache we consider the number of total L3 cache accesses (regardless of whether they are hits or misses) over time as a good indicator of correlation. While the number of total L3 cache accesses only depend on the behavior of the process, the number of hits and misses change according to the key bits being processed, which can change, and are influenced by other processes using the cache; therefore they are not a reliable indicator of correlation.

Although our experiments did not show any false positives we devised two more methods, based on machine learning techniques, that operate in a more fine-grained manner and therefore can be used to detect a spy with more confidence.

The second method makes use of a machine learning technique that has recently become widespread: neural networks. Although computationally more expensive to train, neural networks usually give better results than other supervised learning 
techniques [29] and do not require the data to be preprocessed (e.g. apply feature scaling and mean normalization). Even though there exist many other supervised learning techniques, the good results yielded by our neural network convinced us to explore an option based on semi-supervised anomaly detection, another machine learning technique, instead of iterating over multiple supervised learning models.

In anomaly detection we treat the data coming from the spy process as valid samples and data coming from any other process as anomalies or outliers. We were then able to determine whether a process is benign if it is recognized as an anomaly. The downside of using anomaly detection or supervised learning is that there has to exist data that profiles a sample spy process, similarly to anti-virus applications that require a sample of the malware to be able to recognize it. The main difference is that both neural networks and anomaly detection are flexible enough to detect different types of spy processes as long as they behave similarly to the profiled one, although with less confidence as shown in Chapter 4.

\subsection{Correlation based approach}

The intuition is that both processes spend most of their time in a loop where there is a regular access to potentially cached data. Without loss of generality, with regard to the other attacks, let us analyze the Montgomery ladder implementation, in the point scalar multiplication function ec_GF2m_montgomery

_point_multiply of OpenSSL exploited in the second version of Yarom's FLUSH+RELOAD implementation [9].

The function contains a for loop, previously shown in Figure 2.1, in which the ephemeral key (the scalar used in the multiplication) is scanned bit by bit. Depending on the value of the bit a different conditional branch is evaluated at each iteration where the same two functions ( $g f 2 m \_$Madd and $\left.g f 2 m \_M d o u b l e\right)$ are called with the arguments in a different order. This constant-time implementation should ensure that no useful information leaks through time while executing such function.

The spy, though, in this case is able to time the access to the first function in the first branch and to the second function in the second branch. This allows to guess, with high probability, which branch was chosen and, therefore, the value of the last 
bit of the word from Figure 2.1.

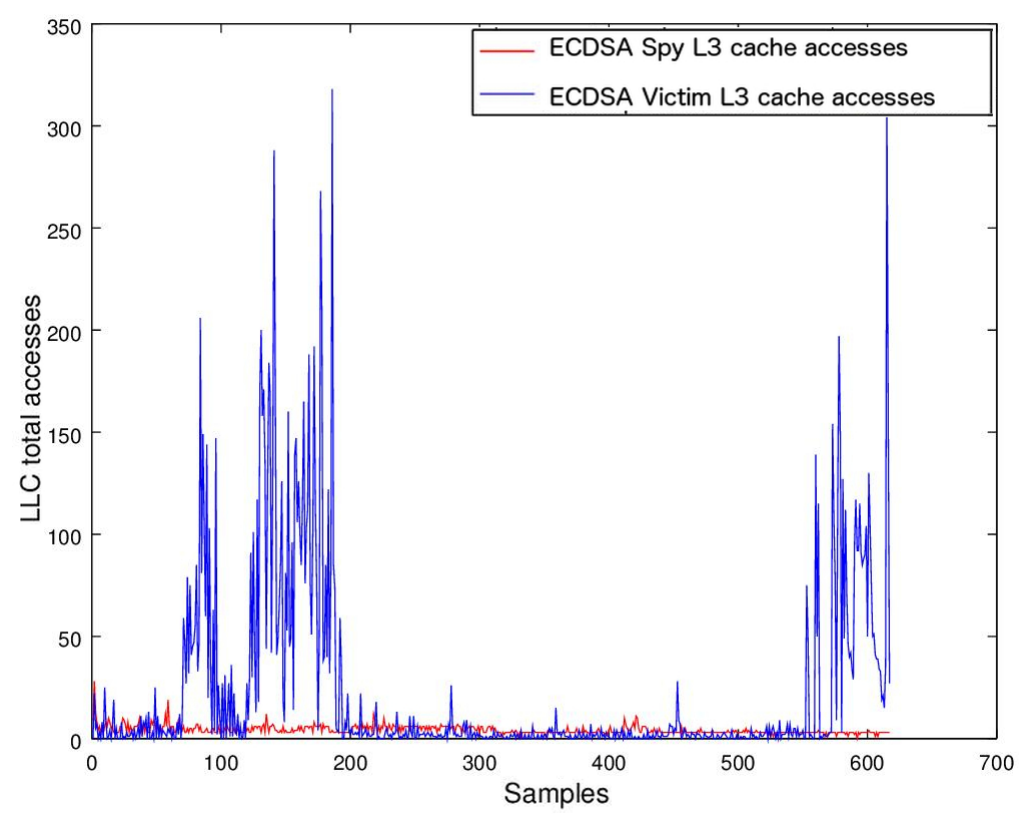

Figure 3.1: Total L3 cache accesses of spy and victim of the attack to ECDSA. The similarities are visible between samples 200 and 550 when the Montgomery ladder loop is executed.

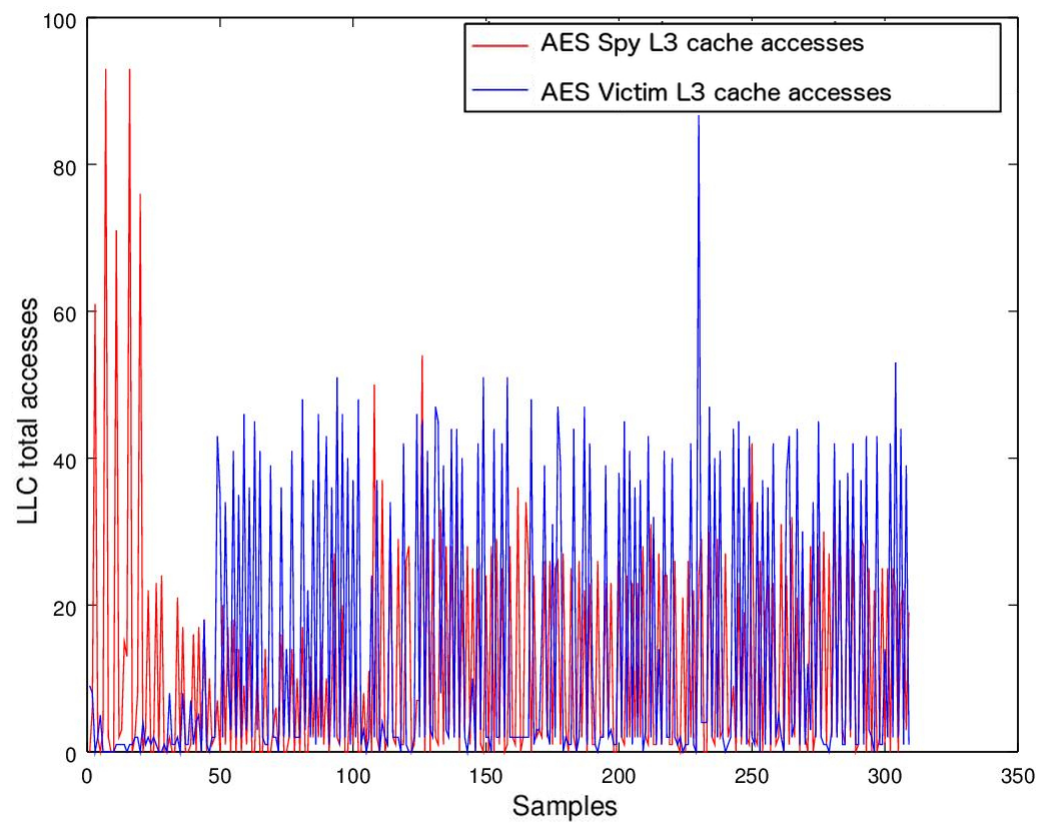

Figure 3.2: Total L3 cache accesses of the spy and the victim of the attack to AES.

Since the loop is executed a large number of times it is fair to presume that its instructions will be loaded in the CPU's cache. In fact, as shown in Figure 3.1, 
between samples 200 and 550, the number of L3 cache accesses over time, while executing the main loop of the Montgomery ladder, is a value oscillating between approximately 0 and 25. The same kind of behavior can be observed, with regard to the AES spy and victim processes, in Figure 3.2 where the L3 cache accesses over time for the two processes, after sample 50, almost overlap.

It is important to note that even though a piece of data is not present in the CPU's cache, each access to it will be registered as an access to the LLC. The MMU will then take care of triggering a cache miss, stall the process and eventually load the necessary data from the main memory into the cache and resume its execution.

Not surprisingly the spy process follows a similar pattern to that of the victim process. The core of the computation lies in a loop where the process continuously flushes and reloads specific addresses from and into the cache. In this case the addresses of interest are the ones of the functions gf2m_Madd and gf 2m_Mdouble.

This regularity is a requirement for the attack to work. In fact, as mentioned in the previous chapter, the spy has to synchronize with the victim to maximize the chances of success.

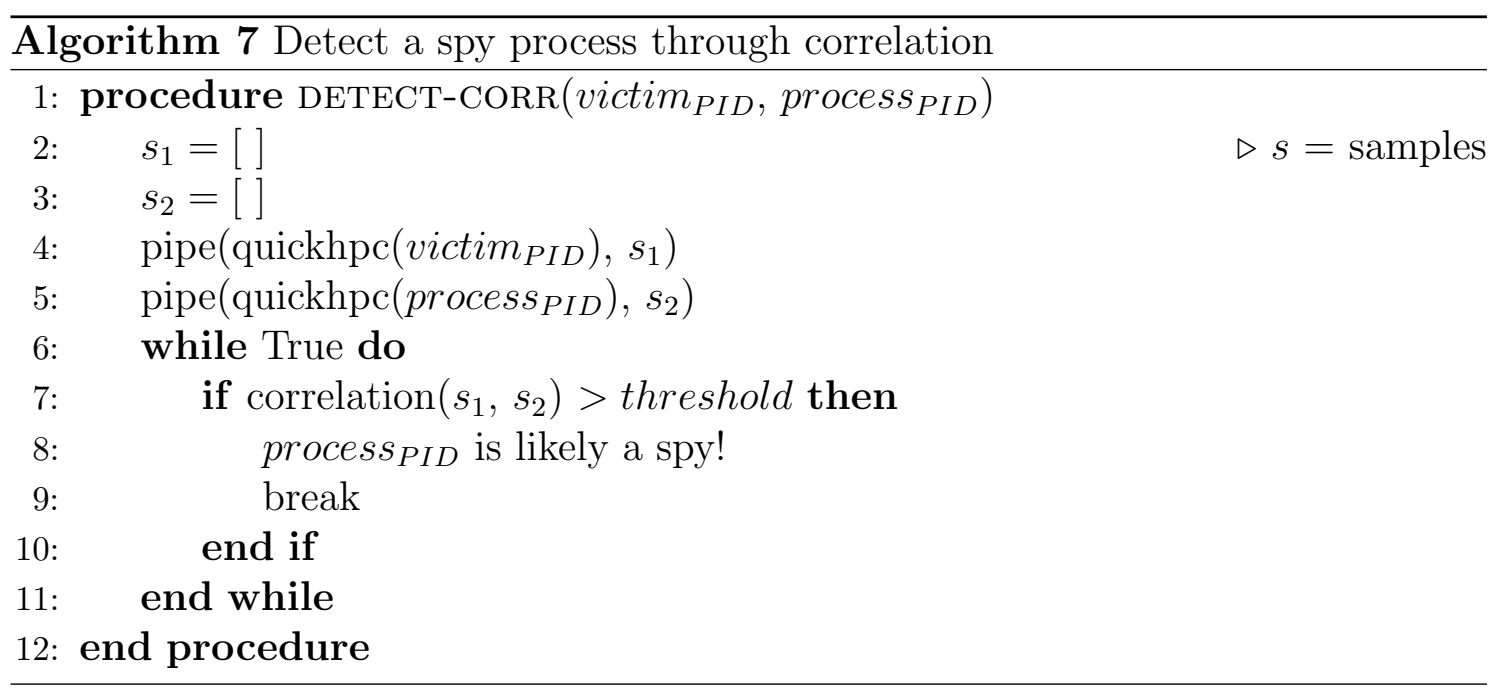

Such behavior can be exploited by monitoring both the victim and the spy at the same time and check how similar the number of LLC accesses over time is, as shown in Algorithm 7. In a real scenario it is often impossible to know when an attack of this sort is in progress therefore it is mandatory to continuously monitor a potential victim process and, separately, each new process spawned by the system.

The variant of the attack by Irazoqui et al., targeting AES, uses a similar mech- 
anism to determine the key used in the last round of an encryption. The substantial difference is that their implementation uses a client-server architecture to trigger the encryptions and repeats the operation thousands of times.

Thanks to the high number of iterations the spy is, in this case, able to retrieve $100 \%$ of the bits of the last round's key. A major drawback of this approach is that it is easy to detect even by using lower resolution tools such as perf-stat. In fact, given the 100 ms minimum resolution of perf-stat and assuming an execution time of 5 seconds, we are able to collect 50 samples, sufficient to determine whether there is correlation or not.

Since only a few milliseconds are sufficient to determine, with high accuracy, whether there exists a correlation between two processes, the monitoring phase does not affect their overall performance. Furthermore, while performing the experiments, it was noticed that the overhead caused by the monitoring tool is negligible.

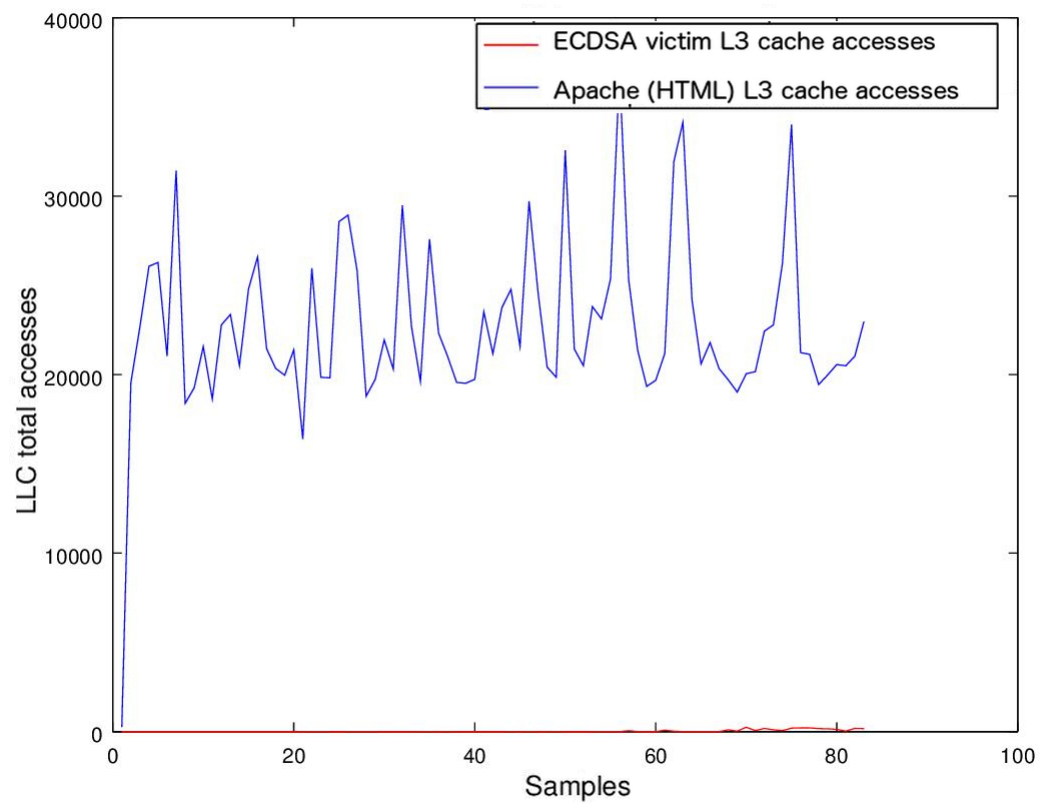

Figure 3.3: Total L3 cache accesses of the Apache webserver serving a 211 B HTML file 1000 times with 100 concurrent clients and the victim of the attack to ECDSA. The difference in cache accesses over time is so high that the line corresponding to the victim process is barely visible. 


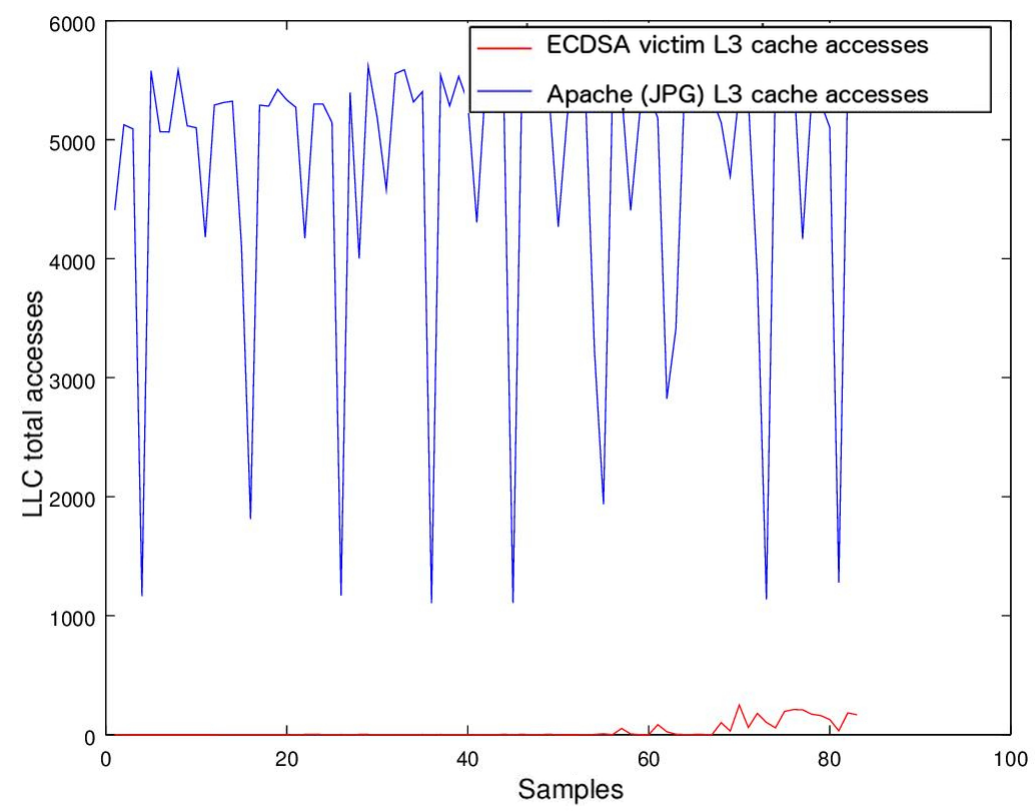

Figure 3.4: Total L3 cache accesses of the Apache webserver serving a 1 MB JPG file 1000 times with 100 concurrent clients and the victim of the attack to ECDSA.

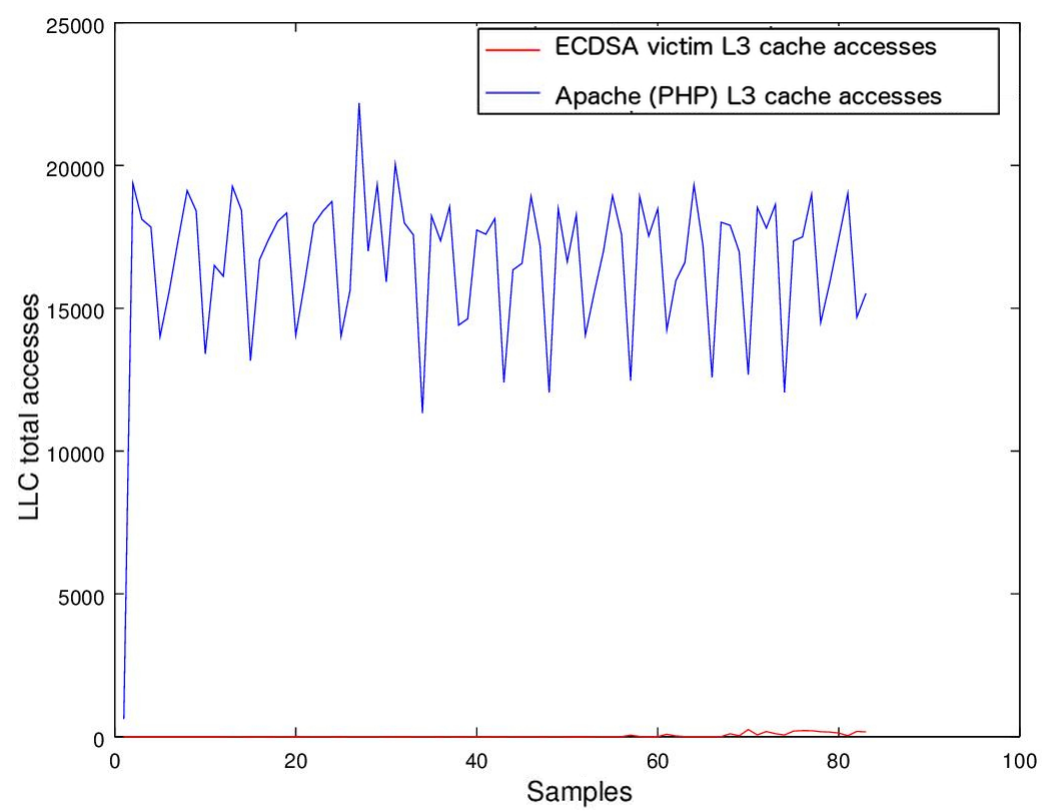

Figure 3.5: Total L3 cache accesses of the Apache webserver serving the output of a PHP script calling php_info 1000 times with 100 concurrent clients and the victim of the attack to ECDSA. The difference in cache accesses over time is so high that the line corresponding to the victim process is barely visible.

Previous Figures 3.1 and 3.2 show how visible this correlation is in both variants while Figures 3.3, 3.4 and 3.5 show how the number of L3 accesses over time differs 
significantly between the tested benign processes and the victim.

\subsection{Anomaly detection based approach}

The reasons why methods based on machine learning techniques might be needed are the potential presence of false positives (that is, there might exist processes that are benign but behave in a similar manner to a spy and would erroneously be flagged as malicious) and due to a more sophisticated spy process which might find a way to escape the detection system based on correlation by creating noise, on purpose, to confuse the detection mechanism (such scenario is discussed further in Chapter 5). Utilizing machine learning techniques allows to profile this behavior as well, increasing the confidence of the detection.

In both methods, based on machine learning, the following combination of events, used as features, yielded the best F-scores: total instructions, total CPU cycles, L2 cache hits, L3 cache misses, L3 cache accesses. Given the good results obtained with this set of features we decided not to explore further combinations and believe this is more a matter of optimization.

By using anomaly detection we can treat the data samples coming from the spy as normal and the data samples coming from other processes as anomalies. Similarly to supervised learning there is a "training" phase where the system is given some samples from the spy process. The training consists of three phases that are repeated until an optimal threshold $\epsilon$ is found:

1. Find $\mu_{j}$ and $\sigma_{j}^{2}$ for each feature $j$.

2. Compute the probability density function $p(x)$ for each sample $x$ and find a value $\epsilon$ such that if $x$ is an anomaly $p(x)<\epsilon$.

3. Test $p(x)$ on a dataset that contains anomalies and verify that such anomalies are recognized. 


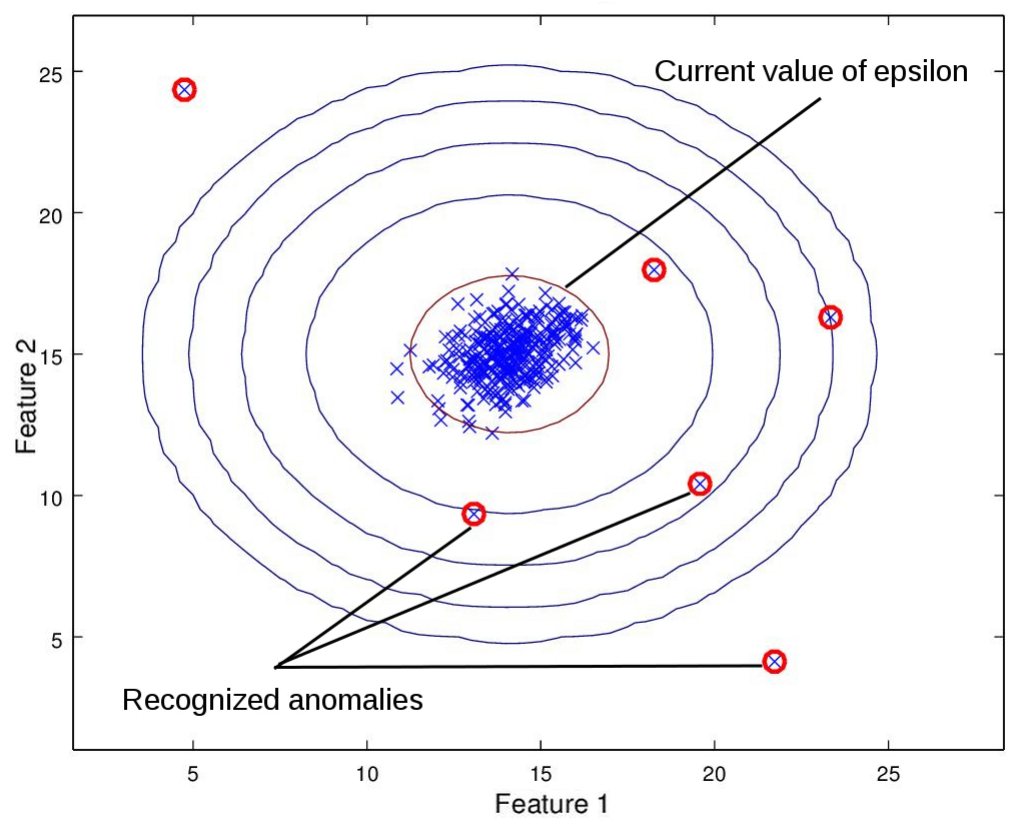

Figure 3.6: In this example different circles representing distinct values of epsilon, the threshold for the density estimation function, visually show how anomalies are flagged according to the value picked.

Figure 3.6 shows a visual example of how changing the value of $\epsilon$ changes which samples are marked as anomalies. The optimal value of epsilon is chosen according to the F-score reached on the cross-validation set at each iteration. Once this phase is complete the system can be used on new data.

\subsection{Based on supervised learning}

Another way of detecting a spy process, by analyzing its behavior at runtime, is to profile it in order to construct some kind of signature that can be used to identify it with a certain confidence, similarly to what anti-virus software does with static signatures.

In the context of supervised learning, the profiling phase translates into a training phase for the classifier (in this case a neural network). The possible outputs represent the two classes of interest: malicious process or benign process. The victim process is always labeled as benign in the training set. The presence of samples from the victim is useful to make the neural network differentiate between two processes that have a very similar behavior (as shown by their correlation) but belong to different 
classes. 


\section{Chapter 4}

\section{Experiments and results}

In this chapter we first introduce an overview of the system used and the results obtained followed by a detailed analysis of such results for each kind of attack.

\subsection{Overview}

All our experiments were performed on an HP Z400 workstation with a Intel Xeon W3670 CPU, operating at a manually fixed clock of $3.2 \mathrm{Ghz}$, and $20 \mathrm{~GB}$ of RAM. The operating system used was Ubuntu 14.04 LTS with kernel Linux 3.13.0-46-generic.

To recreate a realistic environment we simulated workloads, representing the benign processes in the system, that resemble most of the operations performed by the average web backend server nowadays. Different kinds of operations, with different degrees of concurrency, are generated to stress an instance of the Apache web server while serving static and dynamically generated content: a 1KB static HTML file, a 1 MB JPG image and the result of a PHP script that outputs information about the system. This choice was dictated by the fact that this kind of attacks mainly targets cloud computing instances.

For each type of attack we performed 100 iterations where we monitored the spy, the victim and the benign processes operating in different contexts. Each iteration is divided into the following phases:

1. Execution and monitoring of the victim process

2. Execution and monitoring of the spy process 
3. Execution and monitoring of the benign processes

4. Data analysis and prediction

All processes are launched at the same time. Once the spy successfully completes an attack all monitored processes are terminated and the analysis phase begins. During this phase we feed the data to three scripts: one that implements the correlation system, one that implements the anomaly detection system and one that implements the neural network. Each script reports the number of samples used, the confidence of the detection and the time it took to complete it.

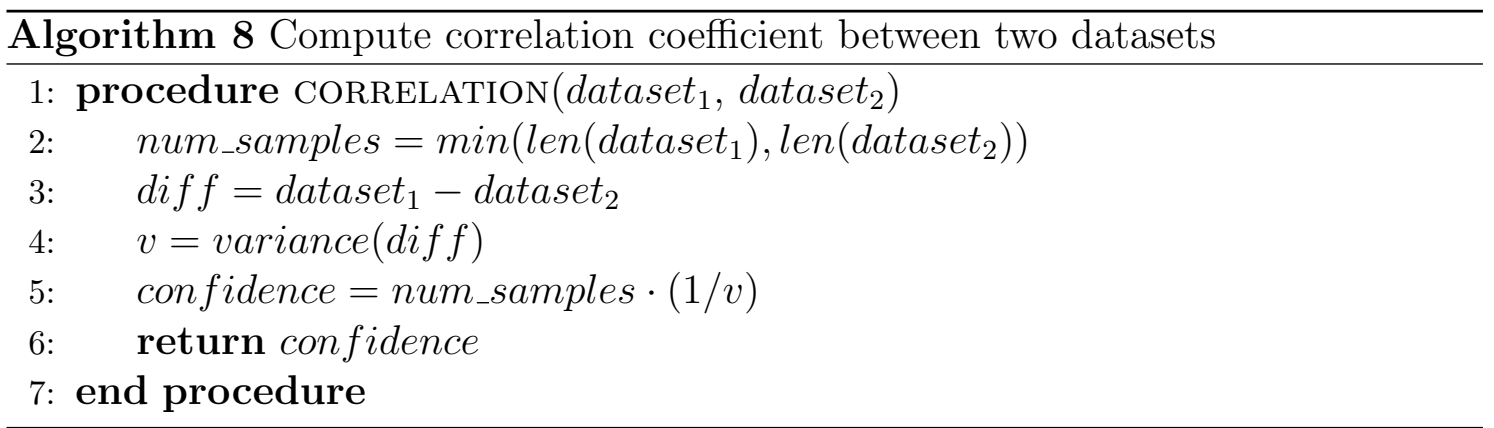

The correlation coefficient is computed as in Algorithm 8. Because we have control over how many samples are collected we assume the number of samples in both datasets is similar. The confidence that a correlation exists is given by the following formula:

$$
\text { confidence }=\text { num_samples } \cdot(1 / \text { variance }) \text {. }
$$

Table 4.1: Benchmarks of the detection method based on correlation

\begin{tabular}{|l|l|l|}
\hline $\begin{array}{l}\text { Correlated processes } \\
\text { (100 iterations) }\end{array}$ & $\begin{array}{l}\text { Min confidence } \\
\text { (samples) }\end{array}$ & $\begin{array}{l}\text { Max confidence } \\
\text { (samples) }\end{array}$ \\
\hline AES spy with AES victim & $0.094715(42)$ & $5.4(522)$ \\
\hline ECDSA spy with ECDSA victim & $0.001565(21)$ & $1.66(744)$ \\
\hline Apache (HTML file) with AES victim & $0.000002(42)$ & $0.000008(157)$ \\
\hline Apache (JPG file) with AES victim & $0.000028(42)$ & $0.000398(862)$ \\
\hline Apache (PHP script) with AES victim & $0.000004(42)$ & $0.000163(862)$ \\
\hline Apache (HTML file) with ECDSA victim & $0.000001(36)$ & $0.000008(157)$ \\
\hline Apache (JPG file) with ECDSA victim & $0.000007(11)$ & $0.000566(1422)$ \\
\hline Apache (PHP script) with ECDSA victim & $0.000002(29)$ & $0.000295(1422)$ \\
\hline Time to find correlation over 500 samples & \multicolumn{2}{|c|}{$0.35 \mathrm{~ms}$} \\
\hline
\end{tabular}

Table 4.1 gives a quantitative insight on how such value changes according to the type of attack we try to detect. With respect to the spy process used, while attacking 
AES the range of confidence varies from a minimum of 0.095 to a maximum of 5.4 but when attacking ECDSA the minimum and maximum confidence values drop to around 0.002 and 1.66 respectively. It is clear that this value is influenced by the number of samples quickhpc was able to process and the higher the number of samples the higher the chance of getting a good level of confidence.

As far as the benign processes are concerned the range decreases significantly with a minimum of $10^{-6}$ and a maximum of $5.66 \cdot 10^{-4}$ which ensure the absence of false positives since the latter value is roughly one order of magnitude lower than the minimum confidence given by any spy process.

Table 4.2: Benchmarks of various operations

\begin{tabular}{|l|l|}
\cline { 2 - 2 } \multicolumn{1}{c|}{} & Time \\
$\begin{array}{l}\text { ECDSA signature } \\
\text { Montgomery Ladder loop }\end{array}$ & $2.8 \mathrm{~ms}$ (default compilation flags) \\
(OpenSS, curve sect571r1) & $9.5 \mathrm{~ms}$ (with debug symbols enabled) \\
\hline $\begin{array}{l}\text { ECDSA signature } \\
\text { Total time } \\
\text { (OpenSSL, curve sect571r1) }\end{array}$ & $\begin{array}{l}6 \mathrm{~ms} \text { (signed 1 B file) } \\
9 \mathrm{~ms} \text { (signed 1 MB file) }\end{array}$ \\
\hline $\begin{array}{l}\text { ECDSA spy } \\
\text { Minimum time needed }\end{array}$ & $\begin{array}{l}2.8 \mathrm{~ms} \text { (the time it takes to complete a } \\
\text { single Montgomery ladder loop) }\end{array}$ \\
\hline $\begin{array}{l}\text { AES spy } \\
\text { Minimum time needed }\end{array}$ & $5 \mathrm{~s}$ (same OS scenario) \\
\hline Maximum quickhpc resolution & $3 \mu s$ (measured with clock_gettime()) \\
\hline
\end{tabular}

The execution time measurements for both the AES and ECDSA victim processes are reported in Table 4.2 where the fastest operation is the execution of the Montgomery Ladder loop, previously shown in Figure 2.1, that takes a maximum of $2.8 \mathrm{~ms}$.

On our system the time to execute Algorithm 8 over a dataset of 500 samples is $0.35 \mathrm{~ms}$ on average. Considering the fastest implementation of the attack has a minimum execution time of $2.8 \mathrm{~ms}$ (i.e. the duration of the Montgomery ladder loop in OpenSSL) there are still $2.45 \mathrm{~ms}$ that can be used to take appropriate countermeasures.

The performance of the neural network is a little worse but good enough for our purposes. Within 0.64 milliseconds the network completes the feedforward propagation over 100 samples and returns the predicted class (spy or not). In this case the confidence is measured as follows: 


$$
\text { confidence }=\text { predictions }_{\text {spy }} / \text { predictions }_{\text {total }}
$$

On the other hand, the anomaly detection system, for a prediction over 100 samples, only takes $0.2 \mathrm{~ms}$ on average making it the fastest one. Unfortunately, it is also the one that suffers the most from noisy data causing it to perform poorly on certain datasets as shown in the next chapter. Even in this case, the confidence is computed with the aforementioned formula.

Table 4.3: Benchmarks of the detection methods based on machine learning techniques

\begin{tabular}{|l|l|l|}
\hline Method & Max F-score & $\begin{array}{l}\text { Time for prediction } \\
\text { (over 100 samples) }\end{array}$ \\
\hline Anomaly detection (AES) & 0.509091 & $0.2 \mathrm{~ms}$ \\
\hline Anomaly detection (ECDSA) & 1.0 & $0.2 \mathrm{~ms}$ \\
\hline Neural network (AES) & 0.932331 & $0.64 \mathrm{~ms}$ \\
\hline Neural network (ECDSA) & 1.0 & $0.64 \mathrm{~ms}$ \\
\hline
\end{tabular}

F-scores for both the anomaly detection system and the neural network are reported in Table 4.3 together with the time it takes to perform a prediction (i.e. to classify) over 100 samples.

\subsection{Detecting AES spy process}

As described in [11] finding the last round's key in an AES encryption, by using a variant of the FLUSH+RELOAD technique, takes a varying amount of time in the order of seconds to minutes.

The execution time depends on the scenario in which the attack is carried out. If both the spy and victim processes are being executed within the same operating system the attacks takes a few seconds (a minimum of $5 \mathrm{~s}$ on our test system) while still being able to recover all the bytes of the key. If the spy and the victim are on separate virtual machines, although share the same CPU, as it often happens with virtualization services such as Digital Ocean [33] or Amazon EC2 [37], the execution takes approximately one minute.

Given the long execution time needed by the spy, to successfully complete an attack, quickhpc is able to collect a very large number of samples in a short amount 
of time. In our experiment we let the spy and the victim run for $50 \mathrm{~ms}, 100$ times less than the minimum time needed to complete the attack.

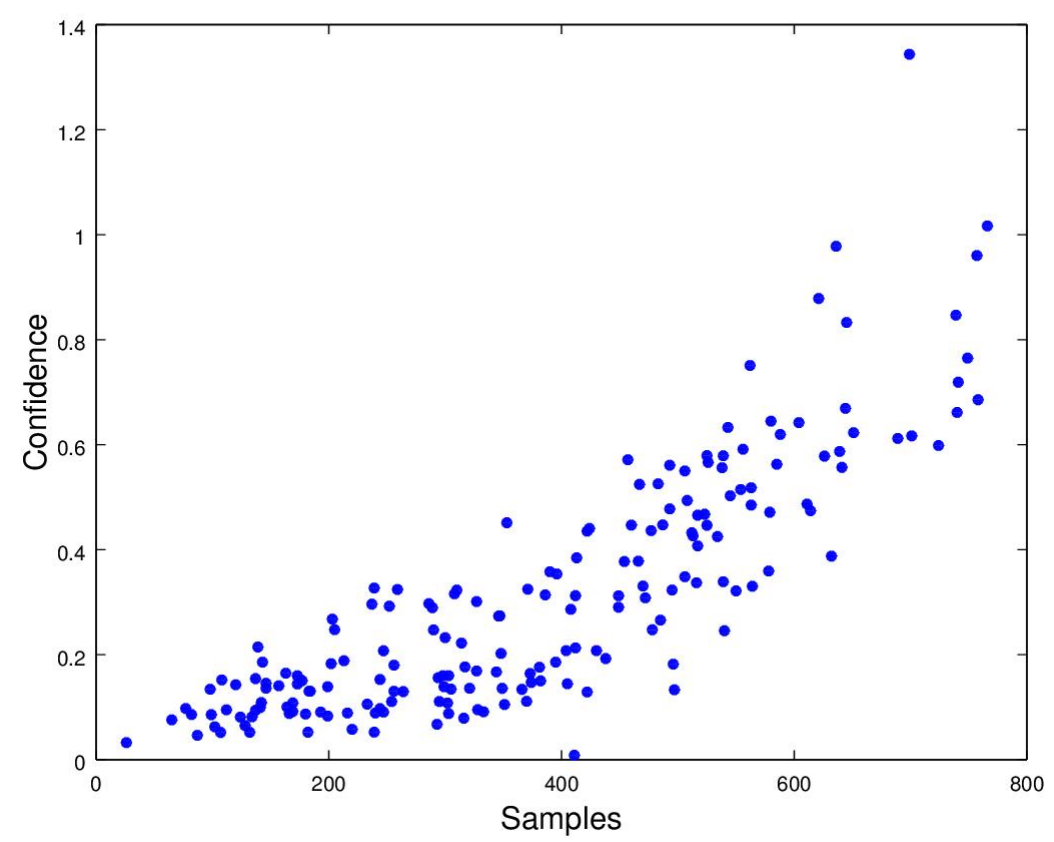

Figure 4.1: Relationship between the number of samples collected during an attack to AES and the confidence of the prediction based on correlation. Even though the relationship is not linear (since the confidence is influenced by noise caused by other processes, scheduling policies etc.) the general trend is that the higher the number of samples the higher the confidence.

Figure 4.1 shows the confidence of the detection according to the number of samples collected. As expected the more the samples the higher the confidence, with the minimum (0.095) reached with 42 samples and the maximum (5.4) reached with 522 samples.

The minimum confidence with regard to the benign processes reached a maximum value of 0.0005 , two orders of magnitude less than the minimum confidence with regard to the spy, effectively eliminating the chance of incurring in false positives in our test environment.

The methods based on machine learning performed very differently in this case. The anomaly detection system performed poorly (see Table 4.3) with a maximum F-score of 0.51 while the neural network reached instead an F-score of 0.93. 


\subsection{Detecting ECDSA spy process}

A complete signature of a $1 \mathrm{~B}$ file, using ECDSA with OpenSSL, takes $6 \mathrm{~ms}$ on average while using a $1 \mathrm{MB}$ file increases this time by $3 \mathrm{~ms}$ for a total of $9 \mathrm{~ms}$.

The main loop used in the Montgomery ladder implementation lasts $2.8 \mathrm{~ms}$ on average which means that, since all detection methods take approximately 0.2 to 0.64 $\mathrm{ms}$, there are around 2-2.5 ms left to take countermeasures, assuming a successful attack is complete once all the bits of the ephemeral key have been scanned.

Considering a resolution of $10 \mu \mathrm{s}$, for quickhpc, we could obtain, in $2.8 \mathrm{~ms}$, roughly 280 samples. The resolution varies according to how the system is performing (i.e. how many processes are running, how the scheduler acts with regard to quickhpc and the monitored process etc.) so the number of samples obtained, and thus the sampling resolution, might be more or less than this theoretical value.

The minimum confidence reached by determining a correlation between the victim and the actual spy was of approximately 0.0016 with 21 samples while the maximum was 1.66 with 744 samples. Even in this case the maximum confidence for the correlation between a benign process and the victim is almost one order of magnitude less than the minimum confidence for the correlation between the spy and the victim.

All machine learning methods, though, performed well with an F-score of 1. Since the time to perform a prediction over 100 samples does not change according to the samples themselves, even in this case it took 0.2 and $0.64 \mathrm{~ms}$ on average respectively for the anomaly detection system and the neural network.

\subsection{Overhead}

We focused on determining the computational overhead caused by quickhpc since it is responsible for collecting the data of the monitored processes. For our system to work in a realistic scenario we need to monitor processes as soon as they start executing and for as long as needed. For example we might modify the operating system so that quickhpc is attached to every new process and keeps monitoring their behavior until they terminate spontaneously or are recognized as suspicious. This mechanism requires quickhpc's overhead to be relatively low. 
We determined the overhead in two different contexts. In the first one we measured the overhead caused by quickhpc with regard to the victim process' alone. That is, whether the execution time of the victim process increased due to the continuous monitoring. In the second one we assessed whether a potentially higher execution time is the consequence of just the higher workload caused by quickhpc or by quickhpc actually interfering with the victim process.

In our first approach we performed 1000 signatures using OpenSSL ECDSA (curve sect571r1), i.e. the victim process described in Chapter 2.4, twice: the first time without monitoring the process and the second time attaching quickhpc. In the worst case the average execution time for the processes that were monitored by quickhpc $(6.16 \mathrm{~ms})$ was $0.99 \%$ higher than the average execution time for the processes that were not monitored $(6.10 \mathrm{~ms})$. To make sure the parallelism offered by a multi-core architecture was not responsible for such low overhead (i.e. because the crypto process and quickhpc were being executed on different cores) we pinned all of the processes to a single core by using the utility taskset [39].

In our second approach we simulated a heavy, CPU-bound, workload by running 2 rounds of 100 instances of the ECDSA victim process. In the first round only the first 99 instances were monitored by quickhpc while in the second round also the last instance was monitored. In both rounds we profiled the last instance to determine its execution time. We then repeated both rounds 1000 times and collected the data to compute the average execution time of the victim process in both cases. The higher workload increased the average execution time of the victim processes, that were not monitored, to $8.45 \mathrm{~ms}$ and the average execution time of the processes monitored by quickhpc to $8.65 \mathrm{~ms}$, i.e. causing an overhead of $2.3 \%$. 


\section{Chapter 5}

\section{A smarter spy process}

The purpose for building a more sophisticated version of a spy process is to evade one or more of the detection systems presented in the previous chapters.

We were able to decrease the confidence range given by the first system, based on correlation, by slightly changing the behavior of the spy so that it would take more time to complete an attack but act in a more clever way. We chose to modify the spy for AES by Irazoqui et al. [11] because of its already long execution time (i.e. minimum $5 \mathrm{~s}$ on our system).

Since the correlation is established only by the total number of cache accesses, the modified spy, similarly to what happens in the actual attack, can start accessing a random number of addresses generating, therefore, a random number of cache hits or misses: accesses nonetheless. 


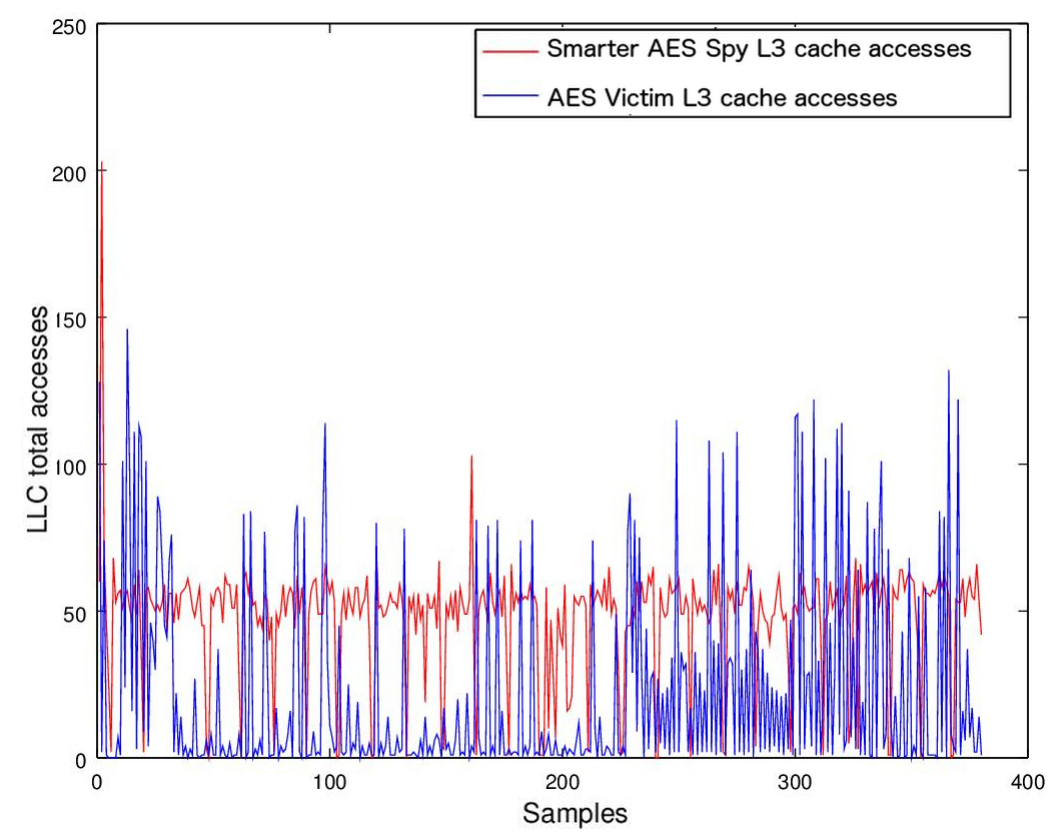

Figure 5.1: Relationship between the total LLC accesses of the AES victim process and the modified version of the spy process.

Although these random accesses, performed for each iteration of the main loop of the spy, cause the total execution time to increase, the success of the attack is in no way influenced. Figure 5.1 shows the relationship between the total cache accesses of the AES victim and the AES modified spy; a very different pattern than the one previously seen in Figure 3.2 with the original spy process.

We modified the spy to access up to 10, 100 and 1000 random addresses for each iteration of the main loop. In all cases the key was correctly retrieved, proving the attack can still be completed, even though the execution time increased dramatically, up to $96 \mathrm{~s}$, in the last case. On the other hand the confidence range of the correlation method noticeably decreased. The minimum value went from 0.095 , for the original spy, to 0.003 for the modified version while the maximum dropped from 5.4 to 0.35 . This proves that it is possible to partially circumvent the detection system based on correlation while still being able to successfully complete an attack.

Table 5.1 shows how the confidence range depends on the number of random addresses used. The data have been collected over 100 attacks for each number of random addresses.

We experienced the opposite trend when trying to catch such process by using the neural network and anomaly detection system. In the first case the maximum 
Table 5.1: Confidence values and execution time for the three variants of the modified version of the AES spy process. Each variant sets a different value for the maximum number of addresses that are probed for each iteration.

\begin{tabular}{|l|l|l|l|}
\hline $\begin{array}{l}\text { Number of } \\
\text { random accesses }\end{array}$ & Min confidence & Max confidence & $\begin{array}{l}\text { Max time } \\
\text { to complete } \\
\text { an attack }\end{array}$ \\
\hline 10 & 0.063545 & 3.197853 & $19 \mathrm{~s}$ \\
\hline 100 & 0.159697 & 1.229513 & $43 \mathrm{~s}$ \\
\hline 1000 & 0.003177 & 0.355517 & $96 \mathrm{~s}$ \\
\hline
\end{tabular}

F-score was 0.98 while in the second case the value dropped to 0.79 , similarly to the unmodified AES spy process. The new behavior clearly makes the process stand out more, rendering the detection even easier when using techniques based on machine learning. 


\section{Chapter 6}

\section{Discussion}

Our results show that it is possible to catch a process that uses the FLUSH+RELOAD technique before the attack can be successfully completed. The fact that our detection system can run as a process in user space makes it convenient to use both on a same-OS scenario and on virtual machines. In the latter scenario the choice would be to either integrate the system into the hypervisor or preinstall the software on any new virtual machine.

In a same-OS scenario the time left between the completion of the detection and the completion of the attack, in the case of the fastest spy where there are 2.6 to $2.2 \mathrm{~ms}$ left, allows for a variety of countermeasures, the simplest being killing the suspicious process and prevent further access to any file or socket opened by it. In case of a cross-VM attack it would be enough, for the hypervisor, to suspend the virtual machine where the spy is running and relocate the one where the victim is running since co-location is the first requirement for these kinds of attacks to work.

The creation of a smarter spy process proved that the detection based on correlation can be partially circumvented opening the doors to further research on how to implement a more advanced variant of the aforementioned attacks. Deceiving the other detection systems, based on machine learning techniques, proved to be a harder task, although the assumption that there exist training data might not always be correct when encountering new variants that work in unexpected ways (which often happens with antivirus software).

The low footprint generated by our system and its ability to run as yet another user space process, together with the fact that most systems are not regularly 
patched against such attacks, make it a good tool for cloud service providers. We think the best way to employ our detection system would be to integrate it in the operating system and attach quickhpc to each new process, monitor them for a predefined amount of time, run one (or all) of the detection algorithms on the collected data and decide whether to terminate the process or simply detach quickhpc and let the process run. 


\section{Chapter 7}

\section{Conclusion and future work}

We introduced three methods to detect a spy process that is performing a cachebased side-channel attack based on techniques such as FLUSH+RELOAD (in general any technique where the attacker accesses the CPU cache with a certain regularity).

While each of the methods has its own strengths and weaknesses we proved that it is definitely feasible to detect and prevent an attack in a relatively short time. Furthermore we did so without altering any of the components of the system (e.g. the kernel) and without causing too much overhead, simply by running our detection system as a user space process.

We are confident that such system might be easily integrated in a physical or virtual cloud environment (such as DigitalOcean or Amazon EC2) either as a separate process (similarly to an anti-virus) or as a plugin for the hypervisor.

On the other hand we also demonstrated how, with just some tweaks, it is possible to deceive one (the simplest) of the detection methods. This, we hope, will fuel more research on increasingly "smarter" detection systems and, consequently, attacks. 


\section{Acknowledgments}

We would like to thank the authors of [9], [10] and [11] for sharing, and assisting us with, the source code of their projects. 


\section{References}

[1] Joan Daemen, Vincent Rijmen. The Design of Rijndael: AES The Advanced Encryption Standard. Springer. ISBN 3-540-42580-2. 2002.

[2] Tian Tian, Chiu-Pi Shih. Software Techniques for Shared-Cache Multi-Core Systems. Intel Developer Zone. 2012.

[3] Andrea Arcangeli, Izik Eidus, Chris Wright. Increasing memory density by using KSM. Red Hat, Inc. 2009.

[4] Ganesh Venkitachalam, Michael Cohen (VMWare, Inc.). Transparent page sharing on commodity operating systems. Patent US7500048 B1. 2009.

[5] Ristenpart, Thomas, et al. Hey, you, get off of my cloud: exploring information leakage in third-party compute clouds.. Proceedings of the 16th ACM conference on Computer and communications security. ACM. 2009.

[6] Mehmet Sinan Inci et al. Seriously, get off my cloud! Cross-VM RSA Key Recovery in a Public Cloud. Cryptology ePrint Archive, Report 2015/898. 2015.

[7] Tsunoo, Yukiyasu, et al. Cryptanalysis of DES implemented on computers with cache. Cryptographic Hardware and Embedded Systems-CHES. 2003.

[8] Osvik, Dag Arne, Adi Shamir, and Eran Tromer. Cache attacks and countermeasures: the case of AES. Topics in CryptologyCT-RSA. 2006.

[9] Yarom, Yuval, and Katrina E. Falkner. Flush+ Reload: a High Resolution, Low Noise, L3 Cache Side-Channel Attack. Proceedings of the 23rd USENIX Security Symposium. USENIX. 2013. 
[10] Yarom, Yuval, and Naomi Benger. Recovering OpenSSL ECDSA Nonces Using the FLUSH+ RELOAD Cache Side-channel Attack. IACR Cryptology ePrint Archive. 2014.

[11] Irazoqui, Gorka, et al. Wait a minute! A fast, Cross-VM attack on AES. Research in Attacks, Intrusions and Defenses. Springer International Publishing. 2014. 299-319.

[12] Gulmezoglu Berk et al. A Faster and More Realistic Flush+ Reload Attack on AES.. 2015.

[13] Irazoqui et al. Know Thy Neighbor: Crypto Library Detection in Cloud. Proceedings on Privacy Enhancing Technologies. 2015.

[14] Sprunt, Brinkley. The basics of performance-monitoring hardware. IEEE Micro 4 pp. 64-71. 2002.

[15] Jonathan Corbet. KSM tries again. LWN. 2009.

[16] Kulah, Yusuf et al. SpyCatcher: Lightweight Online Approaches for Detection of Cache-Based Side Channel Attacks. Manuscript. 2015.

[17] Fei Guo. Understanding Memory Resource Management in VMware vSphere 5.0. VMWare Performance Study. 2011.

[18] Intel. Intel and IA-32 architectures software developers manual. Intel Developer Manuals. 2000.

[19] Anonymous. Security considerations and disallowing inter-Virtual Machine Transparent Page Sharing (2080735) . VMWare knowledge base. 2015.

[20] Salvador Palanca, Stephen A. Fischer, Subramaniam Maiyuran (Intel Corp.). CLFLUSH micro-architectural implementation method and system. Patent US6546462 B1. 2003.

[21] Messerges, Thomas S., Ezzy A. Dabbish, and Robert H. Sloan. Power analysis attacks of modular exponentiation in smartcards. Cryptographic Hardware and Embedded Systems. Springer Berlin Heidelberg, 1999. 
[22] Brown, D. SEC 2: Recommended Elliptic Curve Domain Parameters. 2010.

[23] Joye M., Yen S. M. The Montgomery powering ladder. In Cryptographic Hardware and Embedded Systems-CHES (pp. 291-302). 2002.

[24] Uht, Augustus K., Vijay Sindagi, and Kelley Hall. Disjoint eager execution: An optimal form of speculative execution. Proceedings of the 28th annual international symposium on Microarchitecture. IEEE Computer Society Press. 1995.

[25] Ammons, Glenn, Thomas Ball, and James R. Larus. Exploiting hardware performance counters with flow and context sensitive profiling. ACM Sigplan Notices 32 pp. 85-96. 1997.

[26] de Melo, Arnaldo Carvalho. The new linux perf tools. In Slides from Linux Kongress. 2010.

[27] Zolnowsky, J.E., Whittington, C.L. and Keshlear, W.M., Motorola, Inc. Memory management unit. U.S. Patent 4,473,878. 1984.

[28] Mucci, Philip J., Shirley Browne, Christine Deane, and George Ho. PAPI: A portable interface to hardware performance counters. In Proceedings of the Department of Defense HPCMP Users Group Conference, pp. 7-10. 1999.

[29] Caruana, Rich, and Alexandru Niculescu-Mizil. An empirical comparison of supervised learning algorithms. In Proceedings of the 23rd international conference on Machine learning, pp. 161-168. ACM. 2006.

[30] Uhsadel L., Georges A., Verbauwhede I.. Exploiting Hardware Performance Counters. 5th Workshop on Fault Diagnosis and Tolerance in Cryptography (FDTC). 2008.

[31] Adrian T. et al. Unsupervised Anomaly-based Malware Detection using Hardware Features. 17th International Symposium on Research in Attacks, Intrusions and Defenses (RAID). 2014.

[32] Nishad Herath, Anders Fogh. These are Not Your Grand Daddy's CPU Performance Counters. Black Hat USA. 2015.

[33] Digital Ocean. https://www.digitalocean.com/. Last retrieved: August 2015. 
[34] Van Rijsbergen, C. J. Information Retrieval (2nd ed.). Butterworth. 1979.

[35] Werbos, P.J. Beyond Regression: New Tools for Prediction and Analysis in the Behavioral Sciences.. 1975.

[36] Christopher M. Bishop. Pattern Recognition and Machine Learning, pp. 256269. 2007.

[37] Amazon EC2. https://aws.amazon.com/ec2/. Last retrieved: August 2015.

[38] Kernel Virtual Machine. http://www.linux-kvm.org/page/Main_Page. Last retrieved: August 2015.

[39] Taskset. http://linuxcommand.org/man_pages/taskset1. Last retrieved: August 2015 .

[40] QuickHPC. https://github.com/lambdacomplete/quickhpc. Last retrieved: August 2015. 